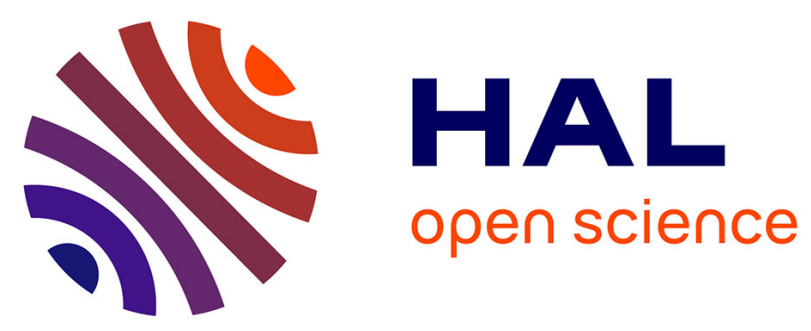

\title{
Linking Theoretical Decision-making Mechanisms in the Simon Task with Electrophysiological Data: A Model-based Neuroscience Study in Humans
}

Mathieu Servant, Corey White, Anna Montagnini, Boris Burle

\section{- To cite this version:}

Mathieu Servant, Corey White, Anna Montagnini, Boris Burle. Linking Theoretical Decision-making Mechanisms in the Simon Task with Electrophysiological Data: A Model-based Neuroscience Study in Humans. Journal of Cognitive Neuroscience, 2016, 28 (10), pp.1501-1521. 10.1162/jocn_a_00989 . hal-01401203

\section{HAL Id: hal-01401203 \\ https://hal-amu.archives-ouvertes.fr/hal-01401203}

Submitted on 23 Nov 2016

HAL is a multi-disciplinary open access archive for the deposit and dissemination of scientific research documents, whether they are published or not. The documents may come from teaching and research institutions in France or abroad, or from public or private research centers.
L'archive ouverte pluridisciplinaire HAL, est destinée au dépôt et à la diffusion de documents scientifiques de niveau recherche, publiés ou non, émanant des établissements d'enseignement et de recherche français ou étrangers, des laboratoires publics ou privés. 


\title{
Linking Theoretical Decision-making Mechanisms in the Simon Task with Electrophysiological Data: A Model-based Neuroscience Study in Humans
}

\author{
Mathieu Servant $^{1,2}$, Corey White ${ }^{3}$, Anna Montagnini ${ }^{1}$, and Borís Burle ${ }^{1}$
}

\begin{abstract}
A current challenge for decision-making research is in extending models of simple decisions to more complex and ecological choice situations. Conflict tasks (e.g., Simon, Stroop, Eriksen flanker) have been the focus of much interest, because they provide a decision-making context representative of everyday life experiences. Modeling efforts have led to an elaborated drift diffusion model for conflict tasks (DMC), which implements a superimposition of automatic and controlled decision activations. The DMC has proven to capture the diversity of behavioral conflict effects across various task contexts. This study
\end{abstract}

\section{INTRODUCTION}

Formal models of perceptual decision-making have traditionally focused on simple two-choice situations. Such models share the assumption that sensory evidence is accumulated until a criterion quantity of evidence is obtained, at which point from where the decision terminates in a choice and motor activity is engaged (Bogacz, Brown, Moehlis, Holmes, \& Cohen, 2006; Ratcliff \& Smith, 2004). However, observers typically face decisions based on multiple streams of sensory information, each of them being associated with multiple response alternatives. Extending decision-making models to more complex and ecological choice situations has recently been the focus of much interest.

Although the majority of studies seeking to address complex decisions have dealt with multiple-choice tasks (e.g., Keuken et al., 2015; Ratcliff \& Starns, 2013; Van Maanen et al., 2012; Leite \& Ratcliff, 2010; Albantakis \& Deco, 2009; Churchland, Kiani, \& Shadlen, 2008; Usher \& McClelland, 2004), others have started to uncover the mechanisms underlying decisions about relevance (Ulrich, Schröter, Leuthold, \& Birngruber, 2015; Servant, Montagnini, \& Burle, 2014; White, Ratcliff, \& Starns, 2011; Hübner, Steinhauser, \& Lehle, 2010). These studies have focused on the conflict paradigm, where task-relevant and taskirrelevant stimulus attributes compete for response acti-

\footnotetext{
${ }^{1}$ Aix-Marseille Université, CNRS, ${ }^{2}$ Vanderbilt University, ${ }^{3}$ Syracuse
} University combined DMC predictions with EEG and EMG measurements to test a set of linking propositions that specify the relationship between theoretical decision-making mechanisms involved in the Simon task and brain activity. Our results are consistent with a representation of the superimposed decision variable in the primary motor cortices. The decision variable was also observed in the EMG activity of response agonist muscles. These findings provide new insight into the neurophysiology of human decision-making. In return, they provide support for the DMC model framework.

vation. In the Simon task, for example, participants are instructed to press a left or right button according to the color of a lateralized stimulus (e.g., left response to a blue target, right response to a red target). Although the location of the stimulus is task irrelevant, it interferes in the decision process: Responses are slower and less accurate when stimulus location and response side do not correspond (incompatible trial) than when they do (compatible trial), a phenomenon known as the "Simon effect" (Hommel, 2011; Simon \& Small, 1969).

Performance in the Simon task is challenging for formal decision-making models for at least two reasons. First, errors are faster than correct responses in the incompatible condition only (Van den Wildenberg et al., 2010). Second, distributional analyses have revealed that the Simon effect decreases as RT increases (Pratte, Rouder, Morey, \& Feng, 2010; Ridderinkhof, 2002), implying that the condition with the longer mean RT also shows the smaller spread of RT (Schwarz \& Miller, 2012). These particular patterns are not naturally predicted by standard decision-making theories (Servant et al., 2014; White et al., 2011; Hübner et al., 2010; Wagenmakers \& Brown, 2007; Luce, 1986). Ulrich et al. (2015) have recently introduced an extension of the drift diffusion model of perceptual decision-making (Ratcliff \& McKoon, 2008; Ratcliff, 1978) able to capture RT distributions and accuracy data in the Simon task, allowing for decomposition and quantification of latent cognitive processes (hereafter referred to as diffusion model for conflict tasks [DMC]). The aim of the present work was to use the DMC as a tool to 
better interpret human electrophysiological measurements in the Simon task. More precisely, we combined DMC predictions with EEG and EMG measurements to test a set of linking propositions (Schall, 2004; Teller, 1984) that specify the relationship between cognitive processes involved in the Simon task and brain activity. We first introduce the DMC architecture and elaborate some linking propositions afterwards.

\section{DMC Architecture}

The standard drift diffusion model assumes continuous and perfect accumulation of the difference between noisy samples of task-relevant sensory information supporting alternative choices. Technically speaking, accumulation grows toward one of two decision termination bounds $b$ (correct decision) and $-b$ (incorrect decision) according to

$$
d x=\mu d t+\sigma d W
$$

where $\mathrm{d} x$ represents the change in accumulated evidence $x$ for a small time interval $d t, \mu$ is the drift rate (i.e., the average increase of evidence in favor of the correct choice per time unit), and $\sigma d W$ denotes Gaussianly distributed white noise (Wiener process) with mean 0 and variance $\sigma^{2} d t$. The DMC preserves this controlled decision-making mechanism (Figure 1, left) but assumes an additional automatic process that operates on taskirrelevant sensory information (e.g., the location of the stimulus in the Simon task). The automatic process briefly spills over to the decision-making process. Decisionmaking is thus determined by superimposed activations of automatic and controlled processes, an architecture reminiscent of a model of attention and automaticity proposed by Logan (1980).
The expected mean of the noisy automatic activation $X_{a}(t)$ is modeled as a scaled gamma function of the following form:

$$
E\left[X_{a}(t)\right]=A e^{-t / \tau}\left[\frac{t e}{(a-1) \tau}\right]^{(a-1)}
$$

where $a$ is the shape parameter of the function and $A$ is its peak amplitude. The peak latency is located at $\tau(a-1), \tau$ being the characteristic time parameter. This pulse-like function represents the short-living contribution of the automatic process to decision-making and favors the correct (incorrect) response in compatible (incompatible) trials (Figure 1, middle). Its first derivative with respect to time is the time-varying drift rate $\mu_{a}(t)$ of the automatic process. Let $\mu_{c}$ denote the (time-constant) drift rate of the controlled process. The time-varying drift rate of the superimposed process is simply the sum of $\mu_{a}(t)$ and $\mu_{c}$ (Figure 1 , right). The model generates a correct (incorrect) response when the superimposed process hits the correct (incorrect) termination bound $b(-b)$. In compatible trials, automatic and controlled processes converge on activation of the correct response, thereby facilitating RT and accuracy. In incompatible trials, the early incorrect automatic activation hampers the decision process and increases the likelihood of an incorrect choice. Ulrich et al. (2015) demonstrated that the DMC captures all the behavioral peculiarities of Simon task performance. Moreover, the model can explain data from other conflict tasks by variations in the characteristic time parameter $\tau$ of the gamma automatic activation.

\section{Linking Propositions}

When perceptual decisions are assigned to particular actions through task instructions, mounting evidence from human neuroimaging and single-unit physiology in behaving monkeys shows that the decision variable is
Figure 1. Architecture of the DMC. The decision process is modeled as the superimposition of automatic and controlled drift diffusion processes. The expected mean (i.e., accumulated drift rate) of each process is illustrated. See text for details. $z=$ starting point of evidence accumulation; $b=$ upper decision termination bound (correct response); $-b=$ lower decision termination bound (incorrect response).

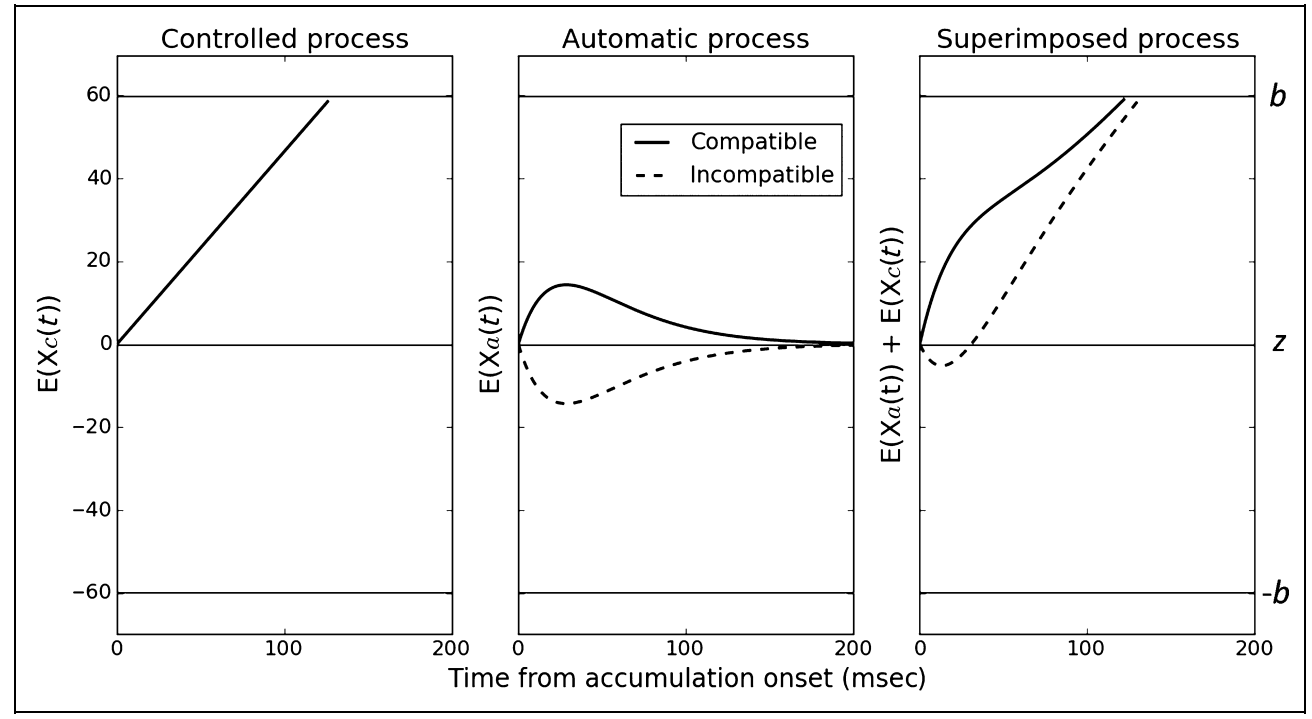




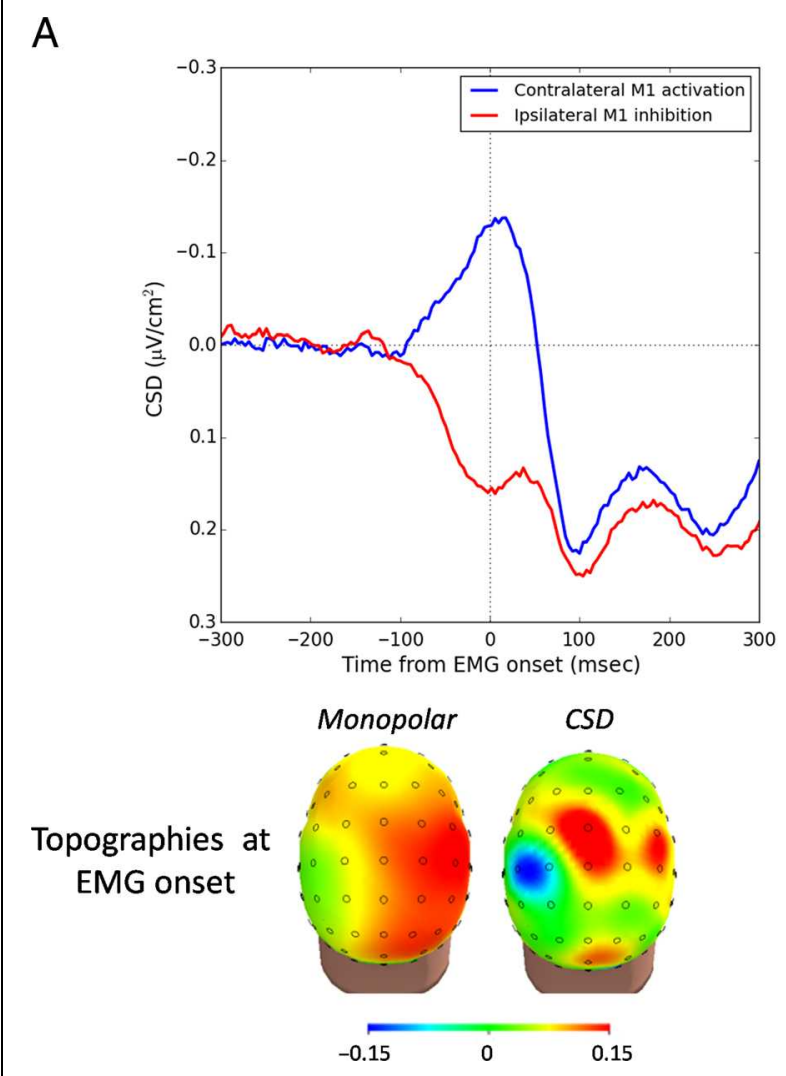

B
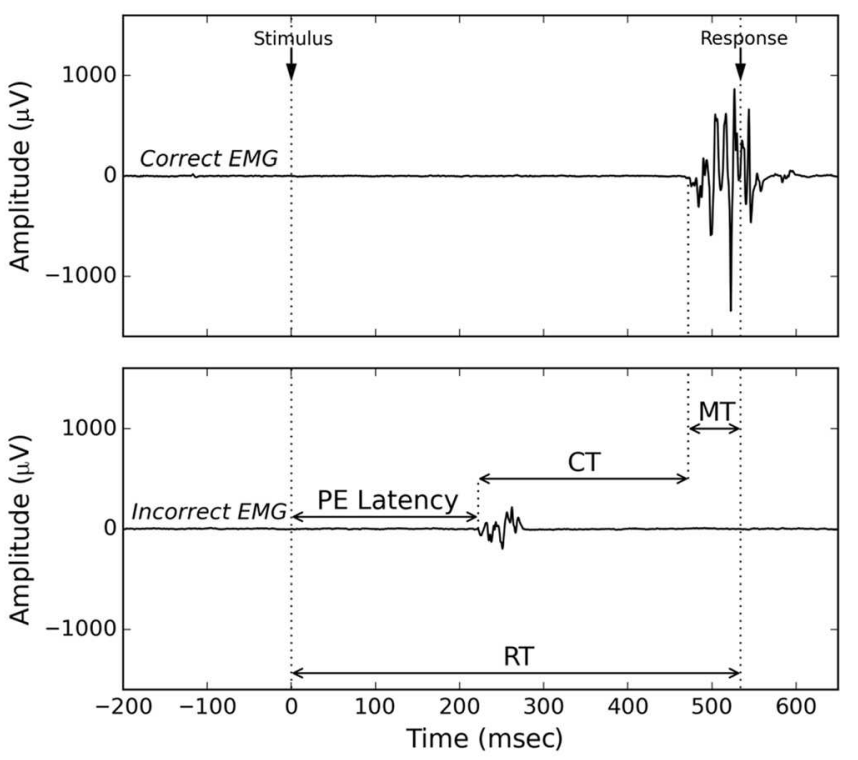

Figure 2. Putative electrophysiological markers of the decision variable. (A) Typical CSD-converted scalp potentials observed over the motor cortices in two-choice tasks involving left and right manual responses (adapted from Vidal et al., 2003). Top: Averaged CSD activities over M1 contralateral (blue trace) and M1 ispsilateral (red trace) to the responding hand time-locked to EMG onset. The contralateral negativity corresponds to an activation of the motor command whereas the ipsilateral negativity corresponds to an inhibition of the nonrequired response. Bottom: Monopolar versus CSD topographic maps at EMG onset. The CSD transformation eliminates blurring effects caused by volume conduction, hence isolating the electrical activity of each motor cortex. (B) EMG activity (in $\mu \mathrm{V}$ ) in the muscles involved (Correct EMG, top) and not involved (Incorrect EMG, bottom) in the required response as a function of time (in $\mathrm{msec}$ ) from stimulus onset. The correct response is preceded by a small EMG burst in the incorrect channel, called a "partial error." RT $=$ RT from stimulus onset to the mechanical response; PE = partial error; CT = correction time (from the incorrect EMG activation to the correct one); MT = motor time (from the correct EMG activation to the correct response).

represented in motor structures that prepare and execute the response (e.g., Kelly \& O'Connell, 2013; O'Connell, Dockree, \& Kelly, 2012; Purcell, Schall, Logan, \& Palmeri, 2012; Selen, Shadlen, \& Wolpert, 2012; Purcell et al., 2010; Donner, Siegel, Fries, \& Engel, 2009; Ratcliff, Cherian, \& Segraves, 2003; Romo, Hernandez, Zainos, Lemus, \& Brody, 2002; Gold \& Shadlen, 2000; Hanes \& Schall, 1996). These findings suggest a continuous flow of information from decision to motor brain regions (Cisek \& Kalaska, 2010; Gold \& Shadlen, 2007). Recently, Kelly and O'Connell (2013) have argued that the lateralized readiness potential $\left(\mathrm{LRP}^{1}\right)$, an EEG marker of unimanual motor preparation, reflects key properties of the theoretical decision variable. In particular, the LRP exhibits rise-tothreshold dynamics (see also Gratton, Coles, Sirevaag, Eriksen, \& Donchin, 1988), the slope of which scales with the quality of sensory information in a meaningful way. The LRP, however, is blind to the contribution of each motor cortex (M1). A more precise measure can be obtained by applying a current source density (CSD) transformation to EEG signals. CSD refers to a group of mathematical trans- formations that compute estimates of the cortical current flowing radially through the skull (Burle et al., 2015; Kayser \& Tenke, 2015), providing a good approximation of the corticogram (Gevins et al., 1987). Contrary to the LRP, CSD methods allow isolation of the electrical activity from each M1. In two-choice tasks, they have revealed a developing negativity over M1 contralateral to the response along with a developing positivity over M1 ispilateral (Burle, Van den Wildenberg, Spieser, \& Ridderinkhof, in press; Vidal et al., 2011; Meckler et al., 2010; Vidal, Grapperon, Bonnet, \& Hasbroucq, 2003). These components peak around the onset of EMG activity responsible for the response (Figure 2A). Converging results from corticograms, intracortical data and stimulation studies indicate that the contralateral negativity corresponds to an activation of the motor command whereas the ipsilateral positivity corresponds to an inhibition of the nonrequired response (reviewed by Burle, Vidal, Tandonnet, \& Hasbroucq, 2004; see also Meckler et al., 2010). Capitalizing upon these findings, the first linking proposition conjectures that the activation/inhibition EEG pattern 
over M1s is the neural signature of the controlled decision process hypothesized by drift diffusion models. Specifically, activation and inhibition components would reflect accumulated task-relevant sensory evidence for and against the chosen response alternative. To test this hypothesis, we manipulated the quality of the task-relevant color evidence in the Simon task and conducted simultaneous EEG and EMG recordings. If Linking Proposition 1 holds, then rising slopes of activation and inhibition EEG components should decrease as the quality of color evidence decreases and exhibit high anticorrelation.

Electrophysiological findings in the Simon task suggest that the location-based automatic activation reaches the motor system. The LRP usually shows an early positive dip in incompatible trials only, presumably reflecting an activation of the incorrect response (reviewed by Leuthold, 2011). Moreover, EMG recordings have revealed covert incorrect EMG activation ("partial error"; Figure 2B) in a portion of trials where the correct response was finally issued. Partial errors are more frequent in incompatible compared with compatible trials, suggesting that the automatic activation reaches the peripheral muscular level (reviewed by Van den Wildenberg et al., 2010). On the basis of these findings, the second linking proposition expands upon the former in assuming that the activation/inhibition EEG pattern over M1s in the Simon task reflects the superimposed diffusion process posited by DMC. To test this hypothesis, we first fit the DMC to each individual behavioral data set. Best-fitting parameters were then used to compute the time course of the expected mean of the superimposed diffusion process. Finally, the grand-averaged time course of the superimposed process was compared against the grand-averaged time course of the activation/inhibition EEG pattern. A comparison of EEG signals with and without partial error trials was also conducted to gain insight into the contribution of the automatic process.

The final linking proposition builds upon our recent computational work, suggesting that EMG activity in conflict tasks reflects up-to-date information about the decision (Servant, White, Montagnini, \& Burle, 2015). We provide an empirical test of this hypothesis by analyzing how partial errors and correct EMG bursts scale with the quality of task-relevant color evidence and how such scaling relates to the theoretical decision variable of DMC. We finally show how the model can be extended to account for EEG and EMG profiles simultaneously and explore consequences of this extension on decision thresholds.

\section{METHODS}

\section{Participants}

Fourteen students (mean age $=23.5$ years, $S D=3.4$ years, 10 women) from the University of Aix-Marseille participated in the study and were paid $€ 15 / \mathrm{hr}$. They were not aware of the purpose of the experiment and reported to have normal or corrected-to-normal vision and normal color vision. This experiment was approved by the ethical committee of the Aix-Marseille University and by the "Comité de Protection des Personnes Sud Méditerrannée 1" (Approval No. 1041). Participants gave their informed written consent according to the Declaration of Helsinki.

\section{Task Design}

Participants performed a Simon task in which the perceptual saturation (i.e., discriminability) of the task-relevant color was manipulated (Servant et al., 2014; Hommel, 1994). The task was programmed in Python 2.7 (www. python.org), using components of the Psychopy toolbox (Peirce, 2007). Participants were instructed to press a right or left button with the corresponding thumb according to the color (blue or red) of a target circle (radius $=$ $\left.0.32^{\circ}\right)$ centered $1.6^{\circ}$ to the left or right of fixation $\left(0.2^{\circ} \times\right.$ $0.2^{\circ}$ gray cross). Colored circles were presented against a black background. Color saturation was varied along the CIE Lightness Chroma Hue device-independent ${ }^{2}$ colorimetric space (Commission Internationale de l'éclairage, 1976), specifically designed to accurately match color perception. Chroma quantifies the percentage of saturation. Red (hue $=30^{\circ}$ ) and blue (hue $=280^{\circ}$ ) colors always had the same lightness $(L=51)$, corresponding to a luminance of approximately $19 \mathrm{~cd} / \mathrm{m}^{2}$ (the lightness of the gray fixation cross was also adjusted to 51). Three suprathreshold chroma levels (low quality of sensory evidence: $15 \%$, medium quality: $25 \%$, high quality: $60 \%$ ) were chosen to obtain approximately similar mean RT differences between each level pair 15-25\% and 25-60\%. We previously demonstrated that mean RT decreases as a power function of chroma intensity in the Simon task (see Figure 6 in Servant et al., 2014), a phenomenon known as Piéron's law (Piéron, 1913). Color calibration was performed by means of a Brontes colorimeter (Admesy B.V., Ittervoort, the Netherlands). Response buttons were fixed on the top of two plastic cylinders $(3 \mathrm{~cm}$ in diameter, $7 \mathrm{~cm}$ in height, $10 \mathrm{~cm}$ separation between buttons), and button closures were transmitted to the parallel port of the computer to reach high temporal precision.

The experiment took place in a dark and soundshielded Faraday cage. Participants were seated in a comfortable chair $180 \mathrm{~cm}$ in front of a CRT monitor with a refresh rate of $75 \mathrm{~Hz}$. Each trial started with a fixation cross whose duration was a random draw from a uniform distribution bounded at 400 and $1200 \mathrm{msec}$. A target circle was then displayed and remained on-screen until the participant responded, with a maximum duration of 1000 msec. The offset of the stimulus and fixation cross served to indicate to the participants the beginning of the intertrial interval $(1000 \mathrm{msec})$, during which they were allowed to blink. Each trial was defined by a factorial combination of target location (left or right), target hue (red or blue), and chroma (three chroma levels). All types of trials were equiprobable and presented in a pseudorandom order so that first-order compatibility sequences (i.e., compatible-incompatible: CI, CC, IC, II) occurred 
the same number of times. Chroma levels were paired equally often with each of the possible compatibility sequence. Half of the participants gave a right-hand response to a blue target and a left-hand response to a red target. This mapping was reversed for the other half. Participants were instructed to respond as fast and accurately as possible to the color of the target circle. They first performed 96 practice trials and worked through 24 blocks of 96 trials, with a short rest between blocks. Practice trials were excluded from analyses. The experiment lasted approximately $105 \mathrm{~min}$.

\section{DMC Fitting}

The DMC was fit to behavioral data using the method proposed by Hübner (2014). The model was simultaneously fit to the quantiles $(0.1,0.3,0.5,0.7,0.9)$ of the RT distributions of correct responses and to the conditional accuracy functions (CAFs) of each individual data set. ${ }^{3}$ CAFs represent accuracy as a function of time. In the Simon task, the incompatible condition is typically associated with an early drop of accuracy, resulting in a concave CAF shape (see Figure 5A, top). This particular pattern implies that incorrect responses are faster than correct responses. CAFs were constructed by sorting the RT data into five bins of equal size; the proportion of errors in each RT bin was then computed, providing the error data considered in the fitting procedure. Model predictions were obtained by running Monte Carlo simulations with an integration constant $d t=1 \mathrm{msec}$ and a diffusion noise $\sigma$ fixed at 4, similar to Ulrich et al. (2015). Predicted and observed data were compared through a likelihood ratio chi-square statistic, $G^{2}$ :

$$
G^{2}=2\left(\sum_{i=1}^{6} n_{i} \sum_{j=1}^{11} p_{i j} \log \frac{p_{i j}}{\pi_{i j}}\right)
$$

The outer summation over $i$ extends over the three chroma levels in each compatibility condition. $n_{i}$ is the number of valid trials per condition. The inner summation over $j$ extends over the 11 RT bins in each condition (6 bins bounded by RT quantiles of correct trials representing $10 \%, 20 \%, 20 \%, 20 \%, 20 \%, 10 \%$ of the data and 5 CAF bins, each one representing $20 \%$ of the data). $p_{i j}$ and $\pi_{i j}$ are, respectively, the observed and predicted proportions of responses in bin $j$ of condition $i$.

The $G^{2}$ statistic was minimized with a python implementation of the Nelder-Mead SIMPLEX algorithm (optimize. fmin function of the SciPy package; Jones et al., 2001) to obtain best-fitting parameters. Eighty thousand trials were simulated for each condition and minimization cycle. Because SIMPLEX is very sensitive to the initial parametric guess, different sets of initial parameter values were used. These values were taken from uniform distributions $U(a, b)$ centered on best-fitting parameters for the Simon task reported by Ulrich et al. (2015). For each parameter, the bounds $a$ and $b$ of the uniform distribu- tion were chosen to span a large space to reduce the likelihood of reaching a local minimum.

Across-trial variability in starting point (uniformly distributed with range $\sigma_{z}$ ) and nondecision time (normally distributed with mean $\mu_{r}$ and variance $\sigma_{r}$ ) was incorporated. ${ }^{4}$ To account for the color saturation manipulation, the drift rate of the controlled process $\mu_{c}$ was allowed to vary between chroma levels. The first model we fit had 10 free parameters (referred to as Model 1): upper decision termination bound $(b$; lower bound $=-b$ ), shape $(a)$, characteristic time $(\boldsymbol{\tau})$, and peak amplitude (A) of the gamma function, drift rate of the controlled process for $15 \%\left(\mu_{\mathrm{c} 15}\right), 25 \%\left(\mu_{\mathrm{c} 25}\right)$, and $60 \%\left(\mu_{\mathrm{c} 60}\right)$ chroma levels, mean nondecision time $\left(\mu_{r}\right)$, across-trial variability in starting point $\left(\sigma_{z}\right)$, and nondecision time $\left(\sigma_{r}\right)$. Because the leading edge of the observed distribution of correct responses was slightly shifted to the right as chroma decreased, we fit an alternative model that also allowed the mean nondecision time to vary between chroma levels. This alternative model (referred to as Model 2) had thus 13 free parameters. Model selection was performed by computing a BIC statistic that penalizes models according to their number of free parameters $f$ :

$$
B I C=G^{2}+f \log \sum_{i=1}^{6} n_{i}
$$

We found that Model 2 had significantly smaller $G^{2}(M=$ $87.1)$ and $B I C(M=185.8)$ values compared with Model 1 $\left(G^{2}: M=114.2 ; B I C: M=191.4\right), p=.0004$ and $p=.037$, respectively (paired-sample permutation tests based on sampling permutation distribution 5000 times). Consequently, we only report best-fitting parameters and predictions of Model 2 in the following sections.

\section{Electrophysiological Recordings and Signal Processing}

EEG and EMG activities were sampled continuously from 64 scalp locations conforming to the 10-20 positioning system (Chatrian, Lettich, \& Nelson, 1988) using a BioSemi Active II system (BioSemi Instrumentation, Amsterdam, the Netherlands). Sampling rate was $1024 \mathrm{~Hz}$. The EMG was recorded from two electrodes pasted $2 \mathrm{~cm}$ apart on the thenar eminence, above the flexor pollicis brevis. Electrodes for vertical and horizontal electrooculogram were respectively located at the Fp2 and below the right eye, and on each outer canthus.

Electrophysiological data were filtered offline (EEG: high-pass $=0.01 \mathrm{~Hz}$; EMG: high-pass = $10 \mathrm{~Hz}$ ). EEG data were re-referenced to the right mastoid, and EMG electrodes were bipolarly referenced. Ocular artifacts were removed by independent component analysis under EEGLAB (Delorme \& Makeig, 2004). Because the EEG signals of some participants were substantially contaminated by muscular activities, we used a Blind Source Separation Algorithm based on Canonical Correlation Analysis 
(BSSCCA; De Clercq, Vergult, Vanrumste, Van Paesschen, \& Van Huffel, 2006) implemented in the AAR plug-in for EEGLAB to remove EMG artifacts and keep as many trials as possible in our analyses. Basically, the BSSCCA method projects the EEG signals into maximally autocorrelated components. Components with the lowest autocorrelation values are assumed to be related to EMG artifacts and are selected according to their spectral power properties. Components having less than 10 times more average power in the EEG band than in the EMG band were considered EMG-related activities and removed. This criterion provided satisfying results. Remaining artifacts were removed manually (overall, $7.2 \%$ of trials were rejected). CSD was estimated by computing the surface Laplacian (SL) ${ }^{5}$ of brain potentials after spherical spline interpolation, using 3 as the degree of spline and a maximum of $15^{\circ}$ for the Legendre polynomial. SL is directly proportional to the radial current flow at scalp (e.g., Perrin, Bertrand, \& Pernier, 1987).

The onset of EMG activity of both hands was marked manually after visual inspection. This method has proven more efficient than automated algorithms (Van Boxtel, Geraats, Van den Berg-Lenssen, \& Brunia, 1993), particularly for detecting small EMG bursts such as partial errors. The experimenter who processed the EMG signals was unaware of the type of mapping and color saturation the EMG traces corresponded to. Trials were classified "correct" or "error," depending on whether button closure took place in the correct or incorrect EMG channel. Among correct trials, trials in which an incorrect EMG activity preceded the correct one ("partial error trials"; see Figure 2B) were dissociated from "pure correct trials."

\section{Data Analyses}

Unless specified, all statistical analyses were performed by means of repeated-measures ANOVAs. Proportions were arc-sine transformed before being submitted to ANOVA to stabilize their variance (Winer, 1971). Data sphericity was assessed by Mauchly's test (Mauchly, 1940). When sphericity was violated, the statistic epsilon $\varepsilon$ was reported and a Greenhouse-Geisser correction (Greenhouse \& Geisser, 1959) was applied. EEG analyses focused on electrodes at standard sites C3 and C4, located above the motor cortices (Koessler et al., 2009). Similar to previous work (e.g., Vidal et al., 2003, 2011), we found that the left- and right-hand responses elicited symmetrical activities (i.e., the hand factor did not interact with any of our experimental factors in all analyses performed, all $p s>.1)$. Consequently, these activities were merged together. Left (odd) and right (even) electrodes respectively correspond to electrodes contralateral and ipsilateral to the responding hand (arbitrary mapping). Individual data sets were included into EMG analyses when the number of partial errors in each condition exceeded 20. All participants fulfilled this criterion.
Table 1. Error Rates (\%) and Mean RTs (msec) for Each Compatibility Condition and Chroma Level Averaged across Participants

\begin{tabular}{lccr}
\hline & \multicolumn{3}{c}{ Chroma Levels (\%) } \\
\cline { 2 - 4 } Condition & 60 & 25 & 15 \\
\hline Error \% & & & \\
Comp & 3.1 & 3.5 & 7.9 \\
Incomp & 4.7 & 6.8 & 12.2
\end{tabular}

\section{Mean RT}

\begin{tabular}{llll} 
Comp & 455 & 478 & 505 \\
Incomp & 474 & 503 & 527 \\
\hline
\end{tabular}

Comp $=$ compatible trials; Incomp $=$ incompatible trials.

\section{RESULTS}

\section{Behavior}

Anticipations (responses faster than $100 \mathrm{msec}, 0.01 \%$ ) and misses (1.8\%) were discarded from analyses. There was a reliable Simon effect on mean RT and error rates, $F(1,13)=60, p=3 \times 10^{-6}$ and $F(1,13)=8.5, p=.012$, respectively (Table 1$)$. The color saturation manipulation was also effective. Mean RT and error rate increased as chroma decreased, $F(2,26)=169.2, p=10^{-15}$ and $F(2$, 26) $=55.3, p=4 \times 10^{-10}$. The interaction between S-R correspondence and chroma on mean RT was not significant, $F(2,26)=1.6, p=.22$, but reached significance on error rates, $F(2,26)=3.6, p=.042$.

\section{Linking Proposition 1}

Figure 3A displays the grand-averaged SL time courses over M1 contralateral (top) and M1 ipsilateral (bottom) to the responding hand in the six conditions for all correct trials. Signals are time-locked to the onset of the stimulus and baseline-corrected -100 to $0 \mathrm{msec}$ before the stimulus-locking event. Anticorrelated ramping activities start to develop around $180 \mathrm{msec}$, betraying the contralateral activation (negativity)/ipsilateral inhibition (positivity) pattern highlighted by previous work (e.g., Vidal et al., 2003, 2011; Meckler et al., 2010). Our first linking proposition conjectures that this pattern is the neural signature of the controlled decision process hypothesized by drift diffusion models. To test this hypothesis, we estimated activation and inhibition ramping slopes by linear regression in a 100-msec window starting $180 \mathrm{msec}$ after stimulus onset (Figure 3A, second shaded area) for each participant and condition and submitted them to an ANOVA with M1 hemisphere, Compatibility, and Chroma as factors. The anticorrelation between ramping slopes, however, makes the analysis difficult to interpret. For example, a main effect of Chroma would result in a 
significant Chroma $\times$ M1 hemisphere interaction. To circumvent this problem, we multiplied ipsilateral slopes by -1 . The analysis revealed a main effect of Chroma, $F(2$, 26) $=5.5, p=.01$ (no other effect reached significance). Ramping slopes generally decreased as chroma decreased, whatever the M1 hemisphere. One exception is the ramping slope of the contralateral activation in the compatible/ $15 \%$ chroma condition, slightly higher compared with that of the $25 \%$ level, presumably due to noise.

To examine dynamics of ramping activities in more detail, we performed a distributional analysis. If activation and inhibition components reflect sensory evidence accumulated for and against the chosen response alternative, their rising slope should decrease as RT increases (Hanes \& Schall, 1996). For each participant and condition, single correct trials were sorted by RT and divided into four equal-sized bins. The grand-averaged SL time courses over M1 contralateral and M1 ipsilateral to the responding hand in the six conditions for each RT bin is plotted in Figure 4A. Ramping slopes of activation and inhibition components generally decrease as RT increases. ${ }^{6}$ We estimated activation and inhibition ramping slopes on this grand-averaged data by linear regression in a 100-msec window starting $180 \mathrm{msec}$ after stimulus onset (Figure 4A, shaded area). Estimated slopes are plotted against the mean RT in the corresponding bin in Figure 4B. The correlation between contralateral activation slopes and RT was high, $r=.59, p=.0027$, so was that between ispislateral inhibition slopes and RT, $r=-.91, p=4 \times$ $10^{-10}$, demonstrating a high dependency between M1 ramping dynamics and $\mathrm{RT}$. The anticorrelation between activation and inhibition slopes was also high, $r=-.68$, $p=.0003$. These analyses provide empirical support to the hypothetical link between the activation/inhibition pattern observed over M1s and the controlled drift diffusion process (see Appendix A for further evidence in favor of this hypothesis).

\section{Linking Proposition 2}

The second linking proposition expands upon the former in assuming that the activation/inhibition EEG pattern over M1s in the Simon task reflects the superimposed diffusion process assumed by DMC. To test this hypothesis, we sought to compare the grand-averaged time course of the superimposed process with the grandaveraged time course of the activation/inhibition pattern. Figure 5 summarizes the very good fit of the DMC to

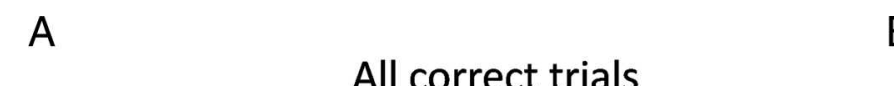

B
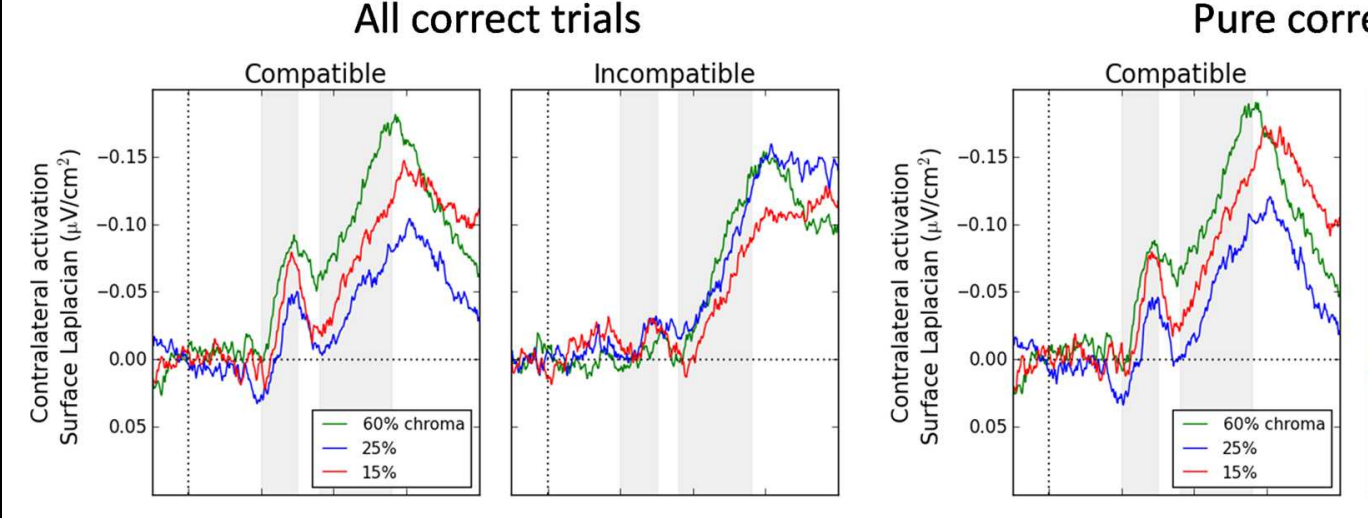

Pure correct trials
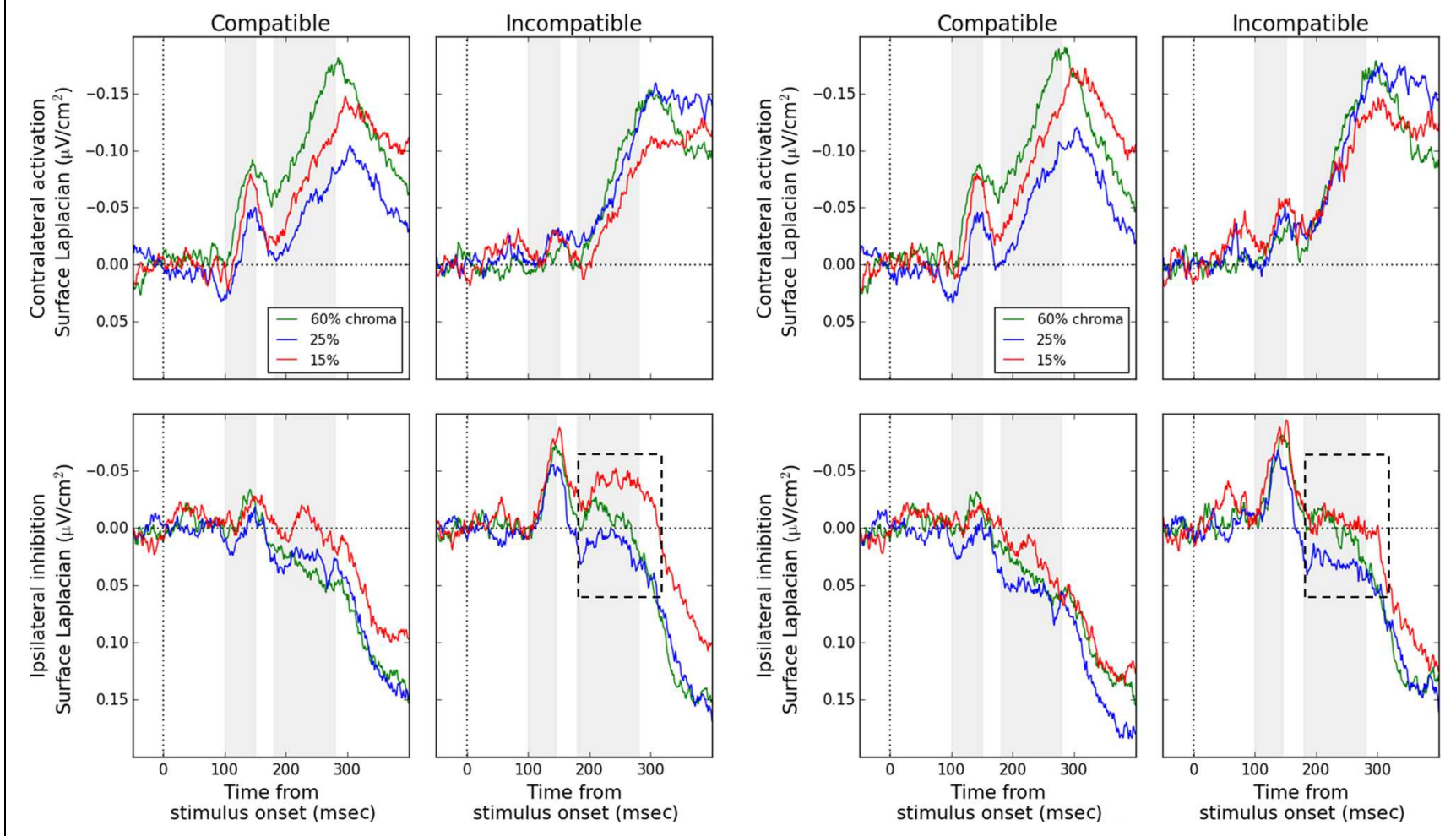

Figure 3. (A) Grand-averaged SL time courses in the six experimental conditions time-locked to stimulus onset in all correct trials. Signals are baseline-corrected -100 to $0 \mathrm{msec}$ before the stimulus-locking event. The top represents SL over M1 contralateral to the responding hand; the bottom represents SL over M1 ispsilateral to the responding hand. Shaded areas illustrate time windows used for analyses. (B) Grand-averaged SL time courses in the six experimental conditions time-locked to stimulus onset in pure correct trials. The removal of partial error trials selectively suppresses the ipsilateral negativity preceding the inhibition ramp in incompatible trials (dashed rectangle). 
behavioral data. Figure 5A displays observed versus predicted CAFs and cumulative density functions (CDFs) of RT for correct responses across experimental conditions averaged over the 14 participants. The model captures accuracy dynamics across conditions and the decrement of the Simon effect as RT increases. Figure 5B shows observed versus predicted RT quantiles of the distributions of correct responses for each individual data set. Data points are gathered around the ideal observed $=$ predicted line, with no systematic deviation. Best-fitting parameters are shown in Table 2. Consistent with Ulrich et al. (2015), we found that the model captures the data with an automatic activation that develops early (i.e., the gamma function peaks at $\tau(a-1)=30$ msec decision time on average). Variations of the drift rate of the controlled process $\mu_{c}$ scaled with the quality of sensory evidence in a meaningful way. Ulrich et al. (2015) demonstrated that variations of $\mu_{c}$ have little impact on the magnitude of the Simon effect, providing an explanation for the observed additive effect of compatibility and chroma on mean RT. Finally, the mean of nondecisional components monotonically increased as chroma decreased, suggesting that a degraded color signal puts higher demands on nondecisional processes such as sensory encoding.

Figure 6 displays the expected mean of the superimposed process computed from best-fitting parameters and averaged across participants. The (1-D) diffusion process was decoupled to illustrate perfectly anticorrelated dynamics of winning and losing accumulators. These theoretical dynamics closely resemble activation and inhibition EEG components (Figure 3A). In particular, the DMC predicts an early incorrect activation in incompatible trials, reflecting the contribution of the automatic process to decision-making. Because automatic and controlled activations are superimposed, the magnitude of this incorrect activation increases as chroma decreases (Figure 6, inset). This prediction was observed in the EEG data. In incompatible trials, the ipsilateral inhibition (positivity) was preceded by a negativity, the amplitude of which increased as chroma decreased (Figure 3A, dashed rectangle). To demonstrate that this early negativity reflects

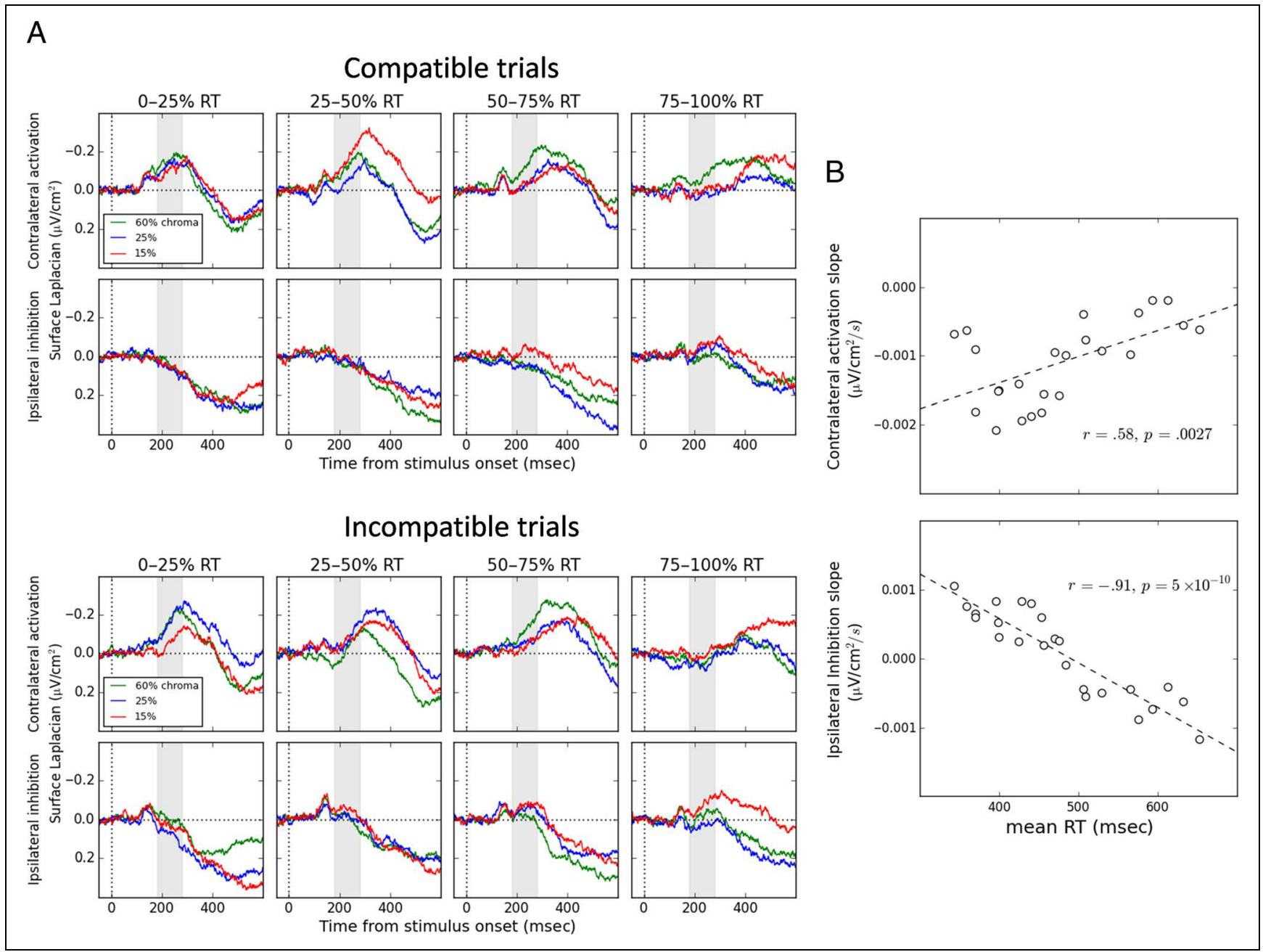

Figure 4. (A) Grand-averaged SL time courses in the six experimental conditions for each of four equal-sized RT bins in all correct trials. Signals are baseline-corrected -100 to $0 \mathrm{msec}$ before the stimulus-locking event. Shaded areas illustrate the time window used to estimate ramping slopes. These slopes are plotted against the mean RT in the corresponding bin in $\mathrm{B}$. 


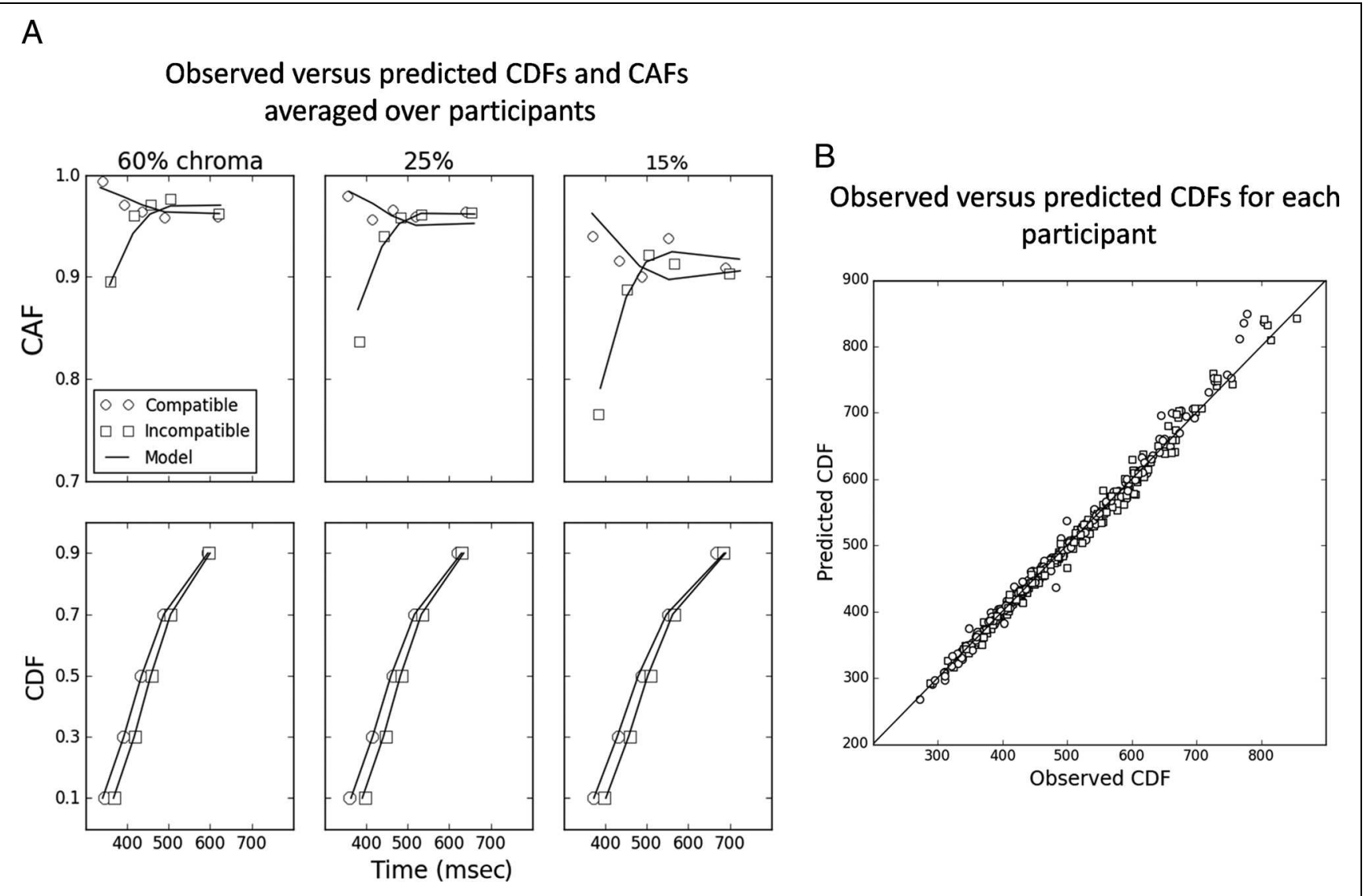

Figure 5. Fit of the DMC to behavioral data. (A) Observed (data points) versus predicted (plain lines) CAF and CDFs of RT for correct responses for each experimental condition. (B) Observed versus predicted CDF quantiles for each condition and individual data set.

the spillover of the automatic process to decision-making, we analyzed the activation/inhibition pattern in pure correct trials (i.e., without EMG partial errors, see Methods). Partial errors in the Simon task are thought to be largely driven by the location-based automatic process (Van den Wildenberg et al., 2010; Burle, Possamai, Vidal, Bonnet, \& Hasbroucq, 2002; Hasbroucq, Possamai, Bonnet, \& Vidal, 1999). Consistent with assumption, we found that the rate of partial errors was generally higher in incompatible compared with compatible trials, $F(1,13)=52.4, p=7 \times 10^{-6}$ (Table 3). Importantly, the rate of partial errors increased as chroma decreased, $F(2,26)=13.3, p=.0001$, the amplitude of the effect being larger in the compatible condition, $F(2,26)=7.8, p=.007, \varepsilon=.72$. Partial error latencies were also affected by chroma, ${ }^{7}$ getting longer as chroma decreased, $F(2,26)=26.7, p=5 \times 10^{-7}$. These results suggest that the automatic process is combined with the controlled process during information processing, consistent with the DMC architecture. Figure 3B displays the grand-averaged SL time courses over M1 contralateral (top) and M1 ipsilateral (bottom) to the responding hand in the six conditions for pure correct trials. Signals are timelocked to the onset of the stimulus and baseline-corrected -100 to 0 msec before the stimulus-locking event, similar to Figure 3A. The removal of partial error trials selectively suppressed the ipsilateral negativity preceding the inhibi-
Table 2. Best-fitting DMC Parameters to Behavioral Data for Each Compatibility Condition and Chroma Level Averaged across Participants

\begin{tabular}{lccc}
\hline \multirow{2}{*}{$\begin{array}{l}\text { DMC } \\
\text { Parameters }\end{array}$} & \multicolumn{3}{c}{ Chroma Levels (\%) } \\
\cline { 2 - 4 }$b$ & $60.5(8.9)$ & & 15 \\
\hline$\mu_{c}$ & $0.465(0.105)$ & $0.430(0.09)$ & $0.326(0.083)$ \\
$A$ & $15.1(2.6)$ & & \\
$a$ & $2.02(0.22)$ & & \\
$\tau$ & $29.4(3)$ & & \\
$\sigma_{z}$ & $18.6(2.9)$ & & \\
$\mu_{r}$ & $334.9(30.8)$ & $355.6(34.5)$ & $358.6(34.7)$ \\
$\sigma_{r}$ & $46.4(13.3)$ & & \\
\hline
\end{tabular}

SDs across participants are shown in parentheses. The DMC was simulated with an integration constant $d t=1 \mathrm{msec}$ and a diffusion coefficient $\sigma=4 . b=$ upper (correct) decision termination bound (incorrect decision termination bound is at $-b) ; \mu_{c}=$ drift rate of the controlled process; $A=$ peak amplitude of the gamma density function (positive in compatible trials, negative in incompatible trials); $a=$ shape of the gamma density function; $\tau=$ characteristic time of the gamma density function; $\sigma_{z}=$ variability in starting point; $\mu_{r}=$ mean nondecision time; $\sigma_{r}=$ variability in nondecision time. 
Figure 6. Expected mean (i.e., accumulated drift rate) of the superimposed diffusion process computed from best-fitting parameters and averaged across participants. The (one-dimensional) diffusion process was decoupled to illustrate perfectly anticorrelated dynamics of winning and losing evidence accumulators. The inset in the losing accumulator of the incompatible condition highlights the amplitude of the early incorrect response activation across chroma levels. Evidence accumulation is preceded by a necessary sensory encoding latency $T e$. $b=$ correct decision termination bound; $z=$ starting point of the accumulation process

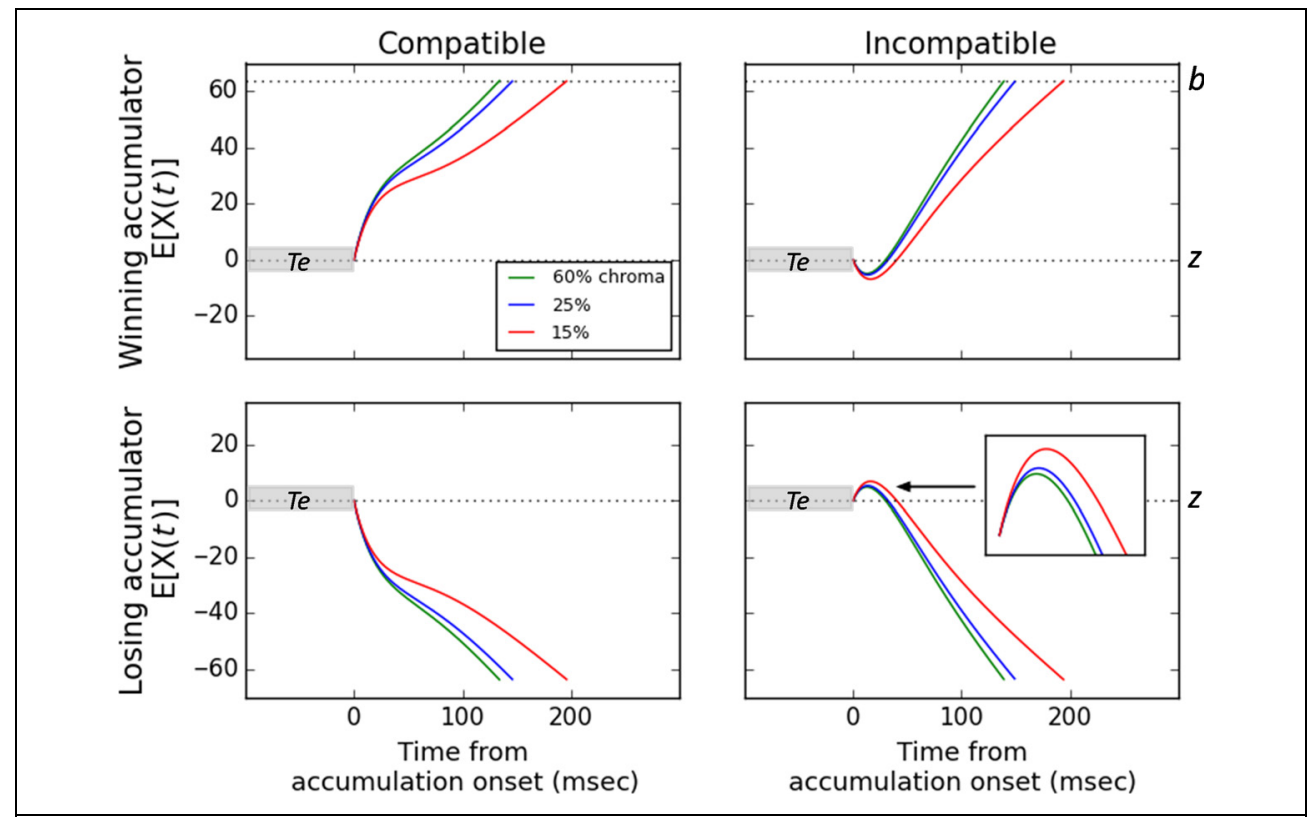

tion ramp in incompatible trials (Figure 3B, dashed rectangle), highlighting the spillover of the automatic process to decision-making. These analyses provide support to the second linking proposition.

Figure 3(A and B) reveals an interesting phenomenon. Developing ramps of activation and inhibition components are preceded by a phasic negative wave peaking at about $150 \mathrm{msec}$ after stimulus onset (hereafter referred to as motor negative MN150). The amplitude of the MN150 appears maximal in the M1 hemisphere contralateral to the stimulus location. One might argue that the MN150 simply reflects a contamination of $\mathrm{C} 3 / \mathrm{C} 4$ electrodes by lateralized visual-evoked potentials through volume conduction effects. The $2-3 \mathrm{~cm}$ spatial resolution of the SL (e.g., Kayser \& Tenke, 2015; Babiloni, Cincotti, Carducci, Rossini, \& Babiloni, 2001) rules out this possibility. Empirical evidence for a motor locus of the MN150 generator is provided in the Appendix B.

We estimated the amplitude of the MN150 for each participant and condition by computing the difference between the mean SL amplitude of two 10 msec time windows centered on the onset (100 msec) and peak latency (150 msec) of the potential (Figure 3A, first shaded area). An ANOVA performed on MN150 amplitudes with M1 hemisphere (contralateral vs. ipsilateral to the responding hand), Compatibility, and Chroma as factors revealed a Compatibility $\times$ Hemisphere interaction, $F(1,13)=16.5$, $p=.0013$ (no other effect reached significance). In compatible trials, the amplitude of the MN150 was larger in M1 contralateral (mean SL amplitude $=-0.075 \mu \mathrm{V} / \mathrm{cm}^{2}$ ) compared with M1 ipsilateral $\left(-0.021 \mu \mathrm{V} / \mathrm{cm}^{2}\right)$ to the responding hand, $F(1,13)=12.9, p=.0033$. The reversed pattern was observed in incompatible trials (mean SL amplitude contralateral $\mathrm{M} 1=-0.020 \mu \mathrm{V} / \mathrm{cm}^{2}$; ipsilateral $\mathrm{M} 1=$ $\left.-0.071 \mu \mathrm{V} / \mathrm{cm}^{2}\right), F(1,13)=17.4, p=.0011$. This analysis shows that the MN150 is maximal in M1 contralateral to the stimulus location, whatever the compatibility condition. It is also invariant to the color saturation manipulation. The MN150 might thus represent the location-based automatic activation before it spills over to the decision process. Although this hypothesis is compatible with the

Table 3. Observed and Predicted Partial Error Rate (\%), Latency (msec), and Correction Time (msec) for Each Compatibility Condition and Chroma Level Averaged across Participants

\begin{tabular}{|c|c|c|c|c|c|c|}
\hline & \multicolumn{6}{|c|}{ Chroma Levels (\%) } \\
\hline & \multicolumn{2}{|c|}{60} & \multicolumn{2}{|c|}{25} & \multicolumn{2}{|c|}{15} \\
\hline & $E M G$ & $D M C$ & $E M G$ & $D M C$ & $E M G$ & $D M C$ \\
\hline \multicolumn{7}{|c|}{ Partial Error Rate } \\
\hline Comp & 11.9 & 8.3 & 14.3 & 9.3 & 18.7 & 12 \\
\hline Incomp & 22.6 & 25.6 & 25.7 & 26.6 & 26.7 & 29 \\
\hline
\end{tabular}

\section{Partial Error Latency}

$\begin{array}{lrrrrrr}\text { Comp } & 277 & 94 & 279 & 98 & 295 & 110 \\ \text { Incomp } & 245 & 39 & 259 & 40 & 280 & 48\end{array}$

Correction Time

\begin{tabular}{lllllll} 
Comp & 136 & 124 & 149 & 128 & 154 & 144 \\
Incomp & 141 & 110 & 153 & 113 & 166 & 127 \\
\hline
\end{tabular}

On average, the best-fitting incorrect EMG bound parameter $(-m)$ of the DMC to partial error rates was $-30.4(S D=8.4)$. Predicted partial error latencies do not contain a sensory encoding time. Predicted partial error latencies and correction times are not a fit to data. Comp $=$ compatible trials; Incomp = incompatible trials. 


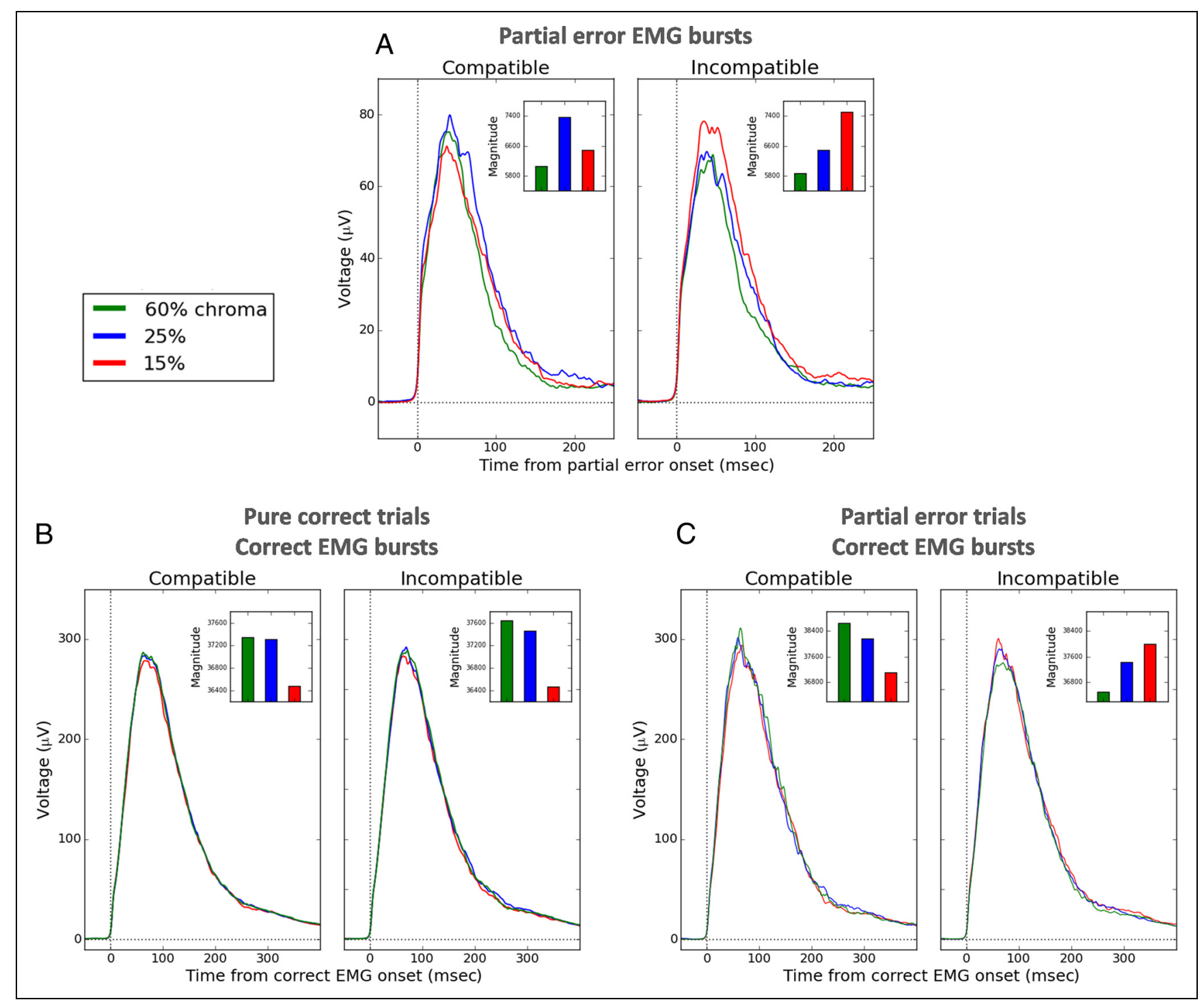

Figure 7. Grand average of rectified EMG activities time-locked to EMG onset of partial errors (A), correct EMG bursts in pure correct trials (B), and correct EMG bursts in partial error trials (C) in the six conditions. Insets represent the grand-averaged magnitude of EMG bursts across chroma levels, computed using the method described in the main text. An 80-Hz low-pass Butterworth filter was applied to signals for display only. Notice that the amplitude of (covert) partial errors is considerably lower than the amplitude of (overt) correct EMG bursts.

DMC framework, it has important limitations. These limitations will be introduced in the Discussion section.

\section{Linking Proposition 3}

The final linking proposition builds upon our recent computational work suggesting that EMG activity in conflict tasks reflects up-to-date information about the decision (Servant et al., 2015). In other words, EMG bursts might be driven by the evolving decision evidence. The observed effects of compatibility and chroma on partial error rates and latencies are consistent with this hypothesis. However, we sought to provide a more direct empirical test by evaluating how the magnitude of EMG bursts scale with chroma and how such scaling relates to the theoretical superimposed diffusion process of the DMC.
Our previous analyses suggest a link between partial errors and the spillover of the automatic process to decisionmaking in incompatible trials. This spillover takes the form of an early incorrect activation, the magnitude of which increases as the task-relevant color evidence decreases (Figure 6, inset). According to the linking proposition at stake, the magnitude of partial errors should increase as chroma decreases in incompatible trials. To test this hypothesis, we estimated the magnitude of partial error EMG bursts for each participant and condition. Partial errors were time-locked to their onset, rectified, baselinecorrected, and averaged. The cumulative sum of the averaged EMG burst was then computed, and the 90th percentile was taken as an estimate of the magnitude. An ANOVA performed on these magnitudes revealed a significant Compatibility $\times$ Chroma interaction, $F(2,26)=4.8$, 
$p=.032, \varepsilon=.7$. In incompatible trials, the magnitude of partial errors monotonically increased as chroma decreased, $F(2,26)=5.5, p=.01$ (Figure 7A). Although a very small trend for a chroma effect showed up in compatible trials $(F(2,26)=2.5, p=.103)$, partial error magnitudes did not meaningfully scale with chroma levels, consistent with the assumption that partial errors are mainly driven by noise or fast guesses in this condition (e.g., Hasbroucq, Burle, Vidal, \& Possamai, 2009; Hasbroucq et al., 1999; Eriksen, Coles, Morris, \& O'Hara, 1985).

Besides partial errors, the third linking proposition makes a particular prediction with regard to the magnitude of (overt) correct EMG bursts. As decision time increases, the drift rate of the superimposed process converges toward that of the controlled process $\mu_{c}$, whatever the compatibility condition (Figure 9A). Because $\mu_{c}$ decreases as chroma decreases, the magnitude of correct EMG bursts should decrease as chroma decreases. Figure 7 (B and C) shows that this is the case, except when the correct EMG burst is preceded by a partial error in the incompatible condition. The magnitude of correct EMG bursts was quantified in the same way as for partial errors. For pure correct trials, the ANOVA revealed a significant effect of Chroma, $F(2,26)=3.2, p=.05$ (Compatibility: $p=$ .54 ; interaction: $p=.83$; Figure $7 \mathrm{~B}$ ). For partial error trials, the ANOVA revealed a trend for a Compatibility $\times$ Chroma interaction, $F(2,26)=2.7, p=.084$ : Although the magnitude of correct EMG bursts in the compatible condition scaled with chroma in the predicted direction, the opposite pattern was observed in the incompatible condition (Figure 7C). This result might suggest a particular partial error correction processing scheme in the incompatible condition.

The hypothetical relationship between the drift rate of the controlled process $\mu_{c}$ and correct EMG bursts can further be assessed by analyzing the motor time
MT (time between the EMG onset of the correct EMG burst and the mechanical button press; see Figure 2B). Specifically, MT should increase when $\mu_{c}$ decreases. We observed this relationship in pure correct trials $(60 \%$ chroma: mean MT = $132.2 \mathrm{msec} ; 25 \%$ : $133.6 \mathrm{msec} ; 15 \%$ : 133.7 msec; main effect of Chroma, $F(2,26)=3.6, p=$ .041 ; Compatibility: $p=.47$; interaction: $p=.65$ ). No significant effect showed up for partial error trials however (all $p s>.1$ ).

These results provide empirical evidence in favor of Linking Proposition 3. They also support the theoretical extension of the drift diffusion model framework proposed in our previous work (Servant et al., 2015). This extension is introduced in Figure 8, with an application to the DMC model. EMG activity is triggered when a decision sample path reaches a level of accumulated evidence $|m|$ inferior to that required for a commitment to a choice, $|m|<|b|$. We refer to $|m|$ as EMG bound and $|b|$ as decision termination bound. This architecture has two important consequences. First, the evidence accumulation decision process carries on even if a motor command has been sent, allowing for potential abortion and correction of this command. Figure 8 illustrates an example of a simulated partial error trial. The model generates a partial error if the decision sample path hits the incorrect EMG bound $-m$ but finally reaches the correct decision termination bound $b$ thanks to timevarying drift rate dynamics. Second, part $^{8}$ of EMG activity directly reflects the evolving decision evidence. In other words, our model extension implements Linking Proposition 3 in the diffusion model framework.

We used the DMC extended with EMG bounds to provide a quantitative account of the EMG data observed in this study. For each individual data set, 80,000 trials per condition were simulated using best-fitting parameters to behavioral data. The incorrect EMG bound $-m$ was fit to
Figure 8. Extension of the drift diffusion model framework proposed by Servant et al. (2015) applied to the DMC. See text for details.

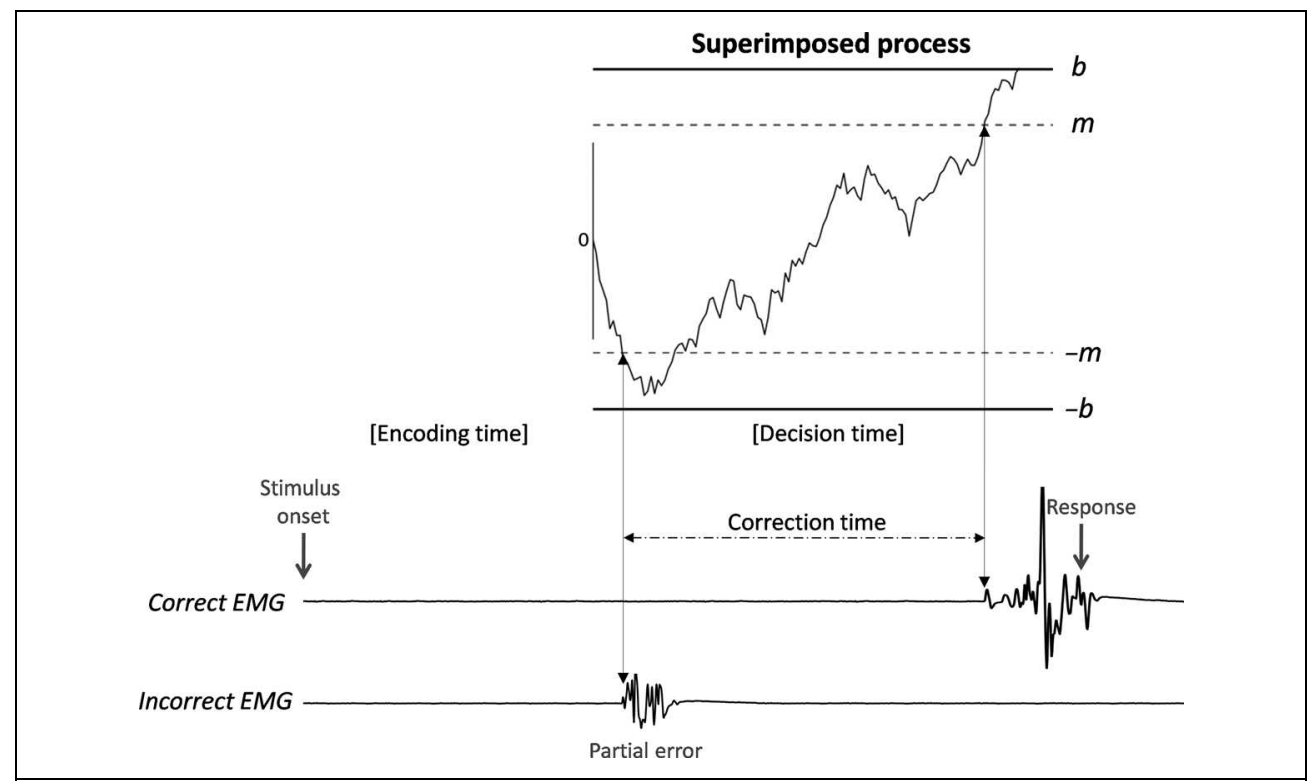


A

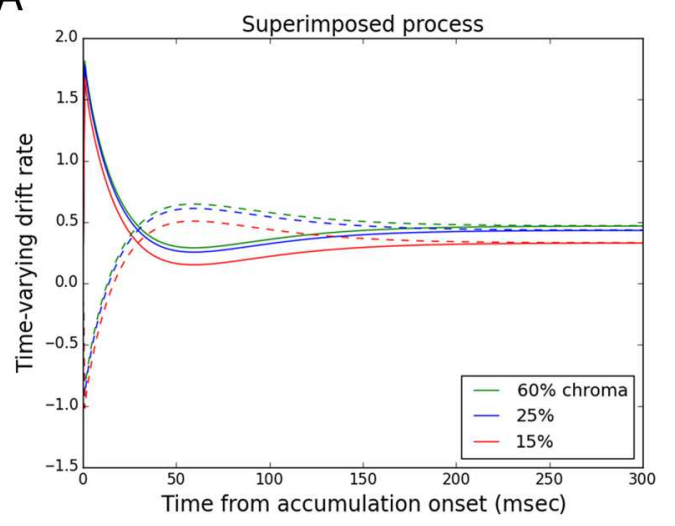

B
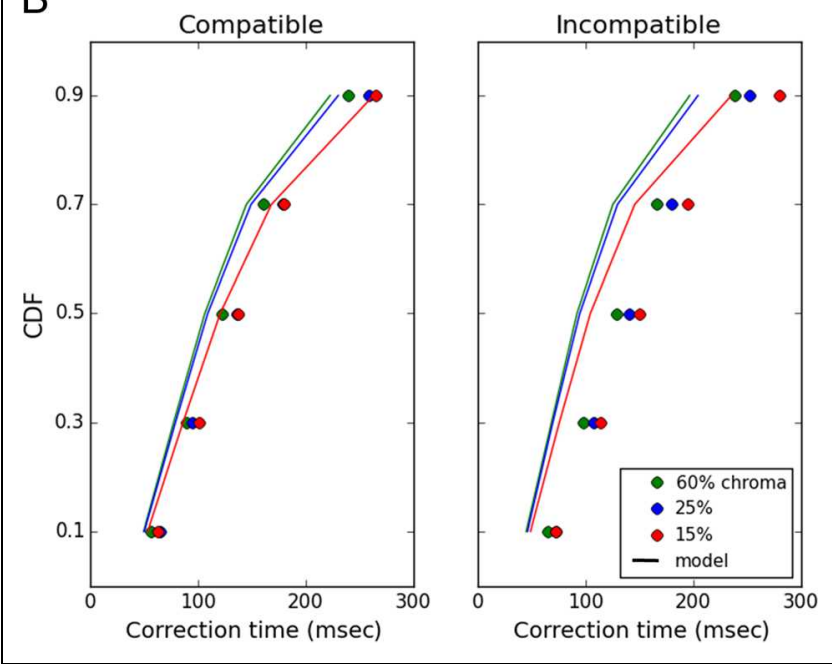

Figure 9. (A) Time-varying drift rate of the superimposed process in the compatible (plain lines) versus incompatible condition (dashed lines) across the three chroma levels, computed from best-fitting parameters and averaged across participants. (B) Observed (data points) versus predicted (plain lines) $\mathrm{CDF}$ of correction times for each condition averaged across participants.

partial error rates by minimizing the following loss function with a Simplex routine:

$$
\text { Loss }=\sum_{i=1}^{6}\left(e_{i}-\varepsilon_{i}\right)^{2}
$$

where $e$ and $\varepsilon$ represent observed and predicted partial error rates for each experimental condition $i$. The parameter $m$ was not allowed to vary between conditions. After this calibration step, the DMC was simulated again to extract predicted partial error latencies and correction times (latency between first hits of the incorrect EMG bound $-m$ and the correct EMG bound $m$; Figure 8 ). These predictions were finally compared against empirical data. ${ }^{9}$ The empirical correction time is defined as the latency between the onset of the partial error and the onset of the correct EMG burst (Figure 2B). Table 3 summarizes the results of the simulations. Although the extended DMC captures compatibility and chroma effects on partial error rates ${ }^{10}\left(p=2 \times 10^{-7}\right.$ and $p=5 \times 10^{-10}$, respectively) and latencies $\left(p=3 \times 10^{-9}\right.$ and $\left.p=2 \times 10^{-9}\right)$, it fails to explain the correction time data. Observed correction times were faster in the compatible compared with the incompatible condition, $F(1,13)=32.8, p=7 \times$ $10^{-5}$, and increased as chroma decreased, $F(2,26)=20.3$, $p=5 \times 10^{-6}$. The DMC, however, predicts faster correction times in the incompatible condition, $F(1,13)=$ $85.1, p=4 \times 10^{-7}$. To understand why, one should compare time-varying drift rate dynamics of the superimposed process in compatible versus incompatible trials (Figure 9A; time-varying drift rate computed from bestfitting parameters and averaged across participants). The plot shows that the Simon effect on mean RT is due to an early decision period (from accumulation onset to $\sim 30 \mathrm{msec}$ ). After $\sim 30 \mathrm{msec}$ decision time, however, the drift rate of the incompatible condition becomes superior to that of the compatible condition. Although such crossing is critical to capture the decrease of the Simon effect over time (Ulrich et al., 2015), it necessarily predicts faster correction times in the incompatible condition, contrary to observed data. To get more insight into this failure, we examined the cumulative distribution of observed versus predicted correction times across conditions, constructed by computing correction time quantiles $(0.1,0.3$, $0.5,0.7,0.9)$. Figure $5 \mathrm{~B}$ shows that the model systematically underestimates each correction time quantile in the incompatible condition by an approximately constant latency, suggesting that a nondecisional processing stage contributes to the correction process. This result echoes our analysis of correct EMG bursts magnitude in partial error trials (Figure $7 \mathrm{C}$ ). The brain might reencode part of the stimulus just after a partial error in incompatible trials. This hypothesis, however, should be taken with caution. Some parameters of the DMC might tradeoff, and we cannot currently exclude that another set of parameters explain the behavioral data equally well and capture the correction time data. Identifying such tradeoffs would require an extensive parameter recovery study.

Our DMC extension makes a strong EEG prediction. Because the contralateral M1 activation of (overt) correct responses reaches the theoretical decision termination bound $b$, it should peak after EMG onset. Partial errors, however, reflect a smaller quantity of accumulated evidence, and their M1 activation should peak earlier, that is, at EMG onset. Figure 10A displays the grand-averaged SL time courses over M1s time-locked to the EMG onset of the correct response in pure correct trials (left), EMG onset of partial errors (middle), and EMG onset of the correct response in partial error trials (right). Signals are baseline-corrected relative to a 2-msec window centered on EMG onset (such that SL amplitude $=0$ at EMG onset) to facilitate comparison with the model predictions. For each participant and condition, we detected the peak latency of the contralateral M1 activation component after application of a $30-\mathrm{Hz}$ low-pass Butterworth filter to minimize spurious effects of noise. One-sample $t$ tests revealed that the contralateral M1 activation of 


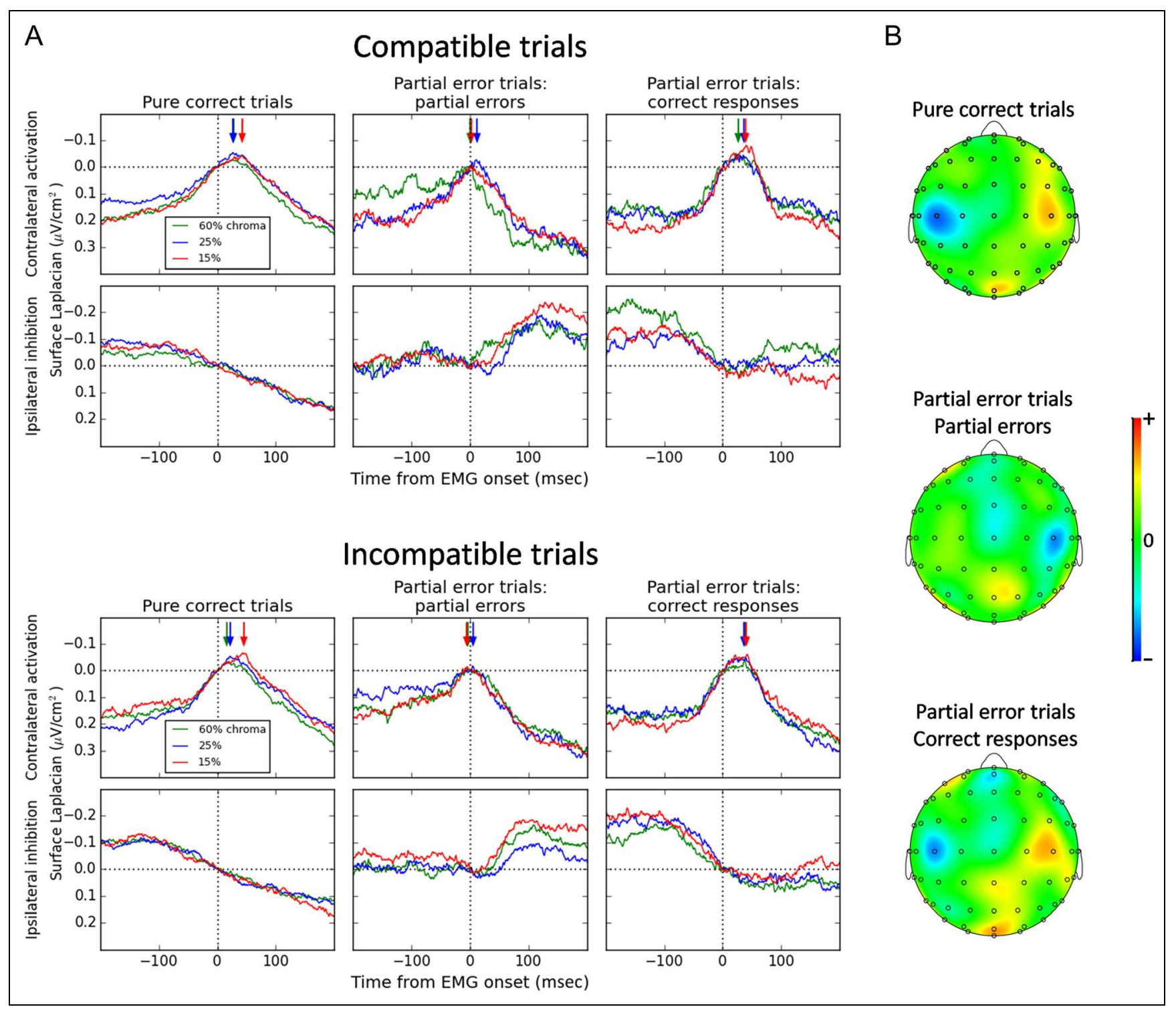

Figure 10. (A) Grand-averaged SL time courses in the six experimental conditions time-locked to EMG onset of correct responses in pure correct trials (left), partial errors (middle), and correct responses in partial error trials (right). Signals are baseline-corrected relative to a 2-msec window centered on EMG onset. Arrows indicate the peak latency of the contralateral activation component (detected after application of a 30-Hz low-pass Butterworth filter to minimize spurious effects of noise) for each condition. (B) SL topographic map at contralateral M1 activation peak latency in the incompatible/15\% chroma condition for each EMG burst category. A different baseline was used to better emphasize the activation/ inhibition pattern.

correct responses peaked significantly after EMG onset, whatever the trial type (all $p s<.01$; see Figure 10A, arrows). The peak latency of the partial error M1 activation did not differ from EMG onset (all $p s>1$ ). These results are consistent with our model extension. SL topographic maps in Figure 9B show that activation and inhibition components are focused over motor cortices, discarding alternative explanations in terms of volume conduction effects.

The alignment of EEG signals to the theoretical EMG bound allows for a final test of our model extension. The peak latency of the contralateral M1 activation of correct responses should increase as chroma decreases, reflecting drift rate dynamics of the controlled process. This pre- diction was verified in pure correct trials (main effect of Chroma: $F(2,26)=3.4, p=.049$; Compatibility: $p=.21$; interaction: $p=.31$; see Figure 10A, arrows). The main effect of Chroma failed to reach significance in partial error trials $(p=.53)$, presumably due to the low signal-to-noise ratio.

\section{DISCUSSION}

This study leveraged DMC predictions and EEG/EMG measurements to elaborate and test a set of linking propositions that specify the relationship between cognitive processes in the Simon task and brain activity. In 
doing so, we sought to enhance our understanding of neurophysiological data. Reciprocally, we sought to better constrain the DMC architecture beyond adequate fits to behavioral data, a method known to have limitations for theory testing (Roberts \& Pashler, 2000). The sections below summarize our main findings and discuss their theoretical consequences.

\section{A Representation of the Superimposed Drift Diffusion Variable in M1s}

Our analyses of SL EEG signals over M1s showed that ramping slopes of activation and inhibition components scale with the quality of task-relevant color evidence and more generally with RT, as expected for a neural integrator (Kelly \& O'Connell, 2013; O'Connell et al., 2012; Donner et al., 2009; Roitman \& Shadlen, 2002; Hanes \& Schall, 1996). The high anticorrelation $(r=-.68)$ observed between activation and inhibition ramps revealed a form of inhibition between competing accumulators, consistent with the general drift diffusion model architecture (Ratcliff \& McKoon, 2008; Ratcliff, 1978). The observed anticorrelation, however, was high but not perfect. The high amount of noise inherent to EEG measurements might have reduced the underlying anticorrelation between neural generators. Perfect anticorrelation might also be implausible from a neurophysiological point of view, due to, for example, ceiling effects of neural firing rates. Consistent with this hypothesis, single-unit recordings of accumulator neurons in behaving monkeys have revealed anticorrelations similar to that reported in this study (reviewed by Gold \& Shadlen, 2007).

Although the drift diffusion model is agnostic to the way inhibition is implemented (Bogacz et al., 2006), empirical evidence suggests that it occurs in a flexible and feedforward manner (i.e., via crossed inputs). The ipsilateral M1 inhibition component is absent when responses are made by two fingers of the same hand (Meynier, Burle, Possamai, Vidal, \& Hasbroucq, 2009) and when no response choice is required (Vidal et al., 2011). Furthermore, functional dissociations have been reported between activation and inhibition components, some manipulations affecting only one of the two (Burle et al., in press; Tandonnet, Burle, Vidal, \& Hasbroucq, 2006). These results speak against an interpretation of inhibition as a passive by-product of reciprocal interhemispheric connections (Ferbert et al., 1992). Two aspects of the present data are consistent with this hypothesis. Figure 10A (left) shows that the ipsilateral M1 inhibition component in pure correct trials carries on even after the contralateral activation peak. Moreover, Figure 9(A and B, middle) suggests a lack of M1 inhibition associated with partial errors. To test this hypothesis, we estimated the slope of the inhibition component by linear regression in a 50-msec time window ending at EMG onset for each participant and condition. Slopes did not differ from 0 in any of the conditions (one-sample $t$ tests, all $p s>.1$ ). ${ }^{11}$ These results highlight the high flexibility of the brain mechanism regulating motor inhibition.

Beyond the relationship between task-relevant sensory information accumulation and activation/inhibition EEG components, we found evidence that the location-based automatic activation spills over to the decision process, as hypothesized by the DMC model (Ulrich et al., 2015). The contribution of the automatic process to decision-making is particularly visible in incompatible trials, where the ipsilateral inhibition ramp is preceded by a short-living negativity (Figure 3A, dashed rectangle). By comparing correct trials with and without EMG partial errors, we were able to unambiguously establish the link between this negativity and the theoretical location-based automatic activation of the incorrect response. Our experimental design allows for a deeper test of the DMC assumption. In incompatible trials, the model assumes that the automatic activation increases as chroma decreases because of the integration of task-relevant and task-irrelevant information into a single decision variable. This prediction was verified in the EEG data. We also found that the rate, latency, and magnitude of partial errors increase as chroma decreases in the incompatible condition. This result provides strong empirical support for the DMC architecture and refutes alternative models in which automatic and controlled processes race independently against each other (see Figure 2 in Van den Wildenberg et al., 2010).

It remains to be determined whether the integration of automatic and controlled processes occurs at the M1 level or in upstream structures. Recent evidence suggests that the decision variable is computed in supramodal brain areas and flows down to the motor system (Kelly \& O'Connell, 2013; O'Connell et al., 2012). These experiments, however, draw upon simple decision tasks and are agnostic with respect to the automatic process. Evidence for a motor locus of the integration might come from the MN150, a phasic negative wave that precedes the activation/inhibition pattern (Figure 3, first shaded area). The amplitude of the MN150 is maximal over M1 contralateral to the stimulus, whatever the compatibility condition, and is not sensitive to chroma. This component might be the neural signature of the location-based automatic activation before it spills over to decision-making. Although this hypothesis is compatible with the DMC framework, it has important limitations. First, one would expect that the Simon effect disappears with vertical stimulus and response arrangements. This has never been observed (e.g., Vallesi, Mapelli, Schiff, Amodio, \& Umilta, 2005; Valle-Inclan \& Redondo, 1998; De Jong, Liang, \& Lauber, 1994). Second, the Simon effect would reverse when the arms are crossed. A regular Simon effect has been systematically documented with this setting (e.g., Hasbroucq et al., 2009; Roswarski \& Proctor, 2003; Wascher, Schatz, Kuder, \& Verleger, 2001; Wallace, 1971). An alternative interpretation of the MN150 comes from a line of EEG research in spatial compatibility tasks showing early lateralized motor deflexions that do not reflect 
movement-related activities. Using an auditory version of the Simon task, Leuthold and Schröter (2006) reported a lateralized frontocentral activity in the time range of the MN150 that was not modulated by the effector condition (hand, foot, eye), presumably reflecting a motor-attentional mechanism (see also Praamstra \& Oostenveld, 2003; Wascher \& Wauschkuhn, 1996). This linking proposition should be tested in future work.

\section{EMG Activity and the Decision Variable}

The final linking proposition conjectures that part of EMG activity reflects the evolving decision variable. Consistent with this proposition, we found that covert and overt EMG bursts scale with the quality of sensory evidence in a way predicted by the superimposed decision variable of DMC. At first sight, this result suggests a continuous flow of the decision variable down to the effectors. The transmission of information from M1 to the flexor pollicis brevis, however, cannot be purely continuous: EMG bursts do not exhibit a ramp-like activity but instead a discrete onset, suggesting that a mechanism gates the flow of information. Incorporation of EMG bounds into the drift diffusion model framework is a simple realization of this idea (Servant et al., 2015). We extended the DMC with EMG bounds and provided a quantitative account of the EMG data. This extension made additional predictions on evidence accumulation threshold dynamics that were verified in the EEG data.

It is unclear whether the link between EMG activity and the decision variable extends beyond conflict tasks. Also, the functional significance of EMG bounds remains to be clarified. We previously hypothesized that the brain might engage motor activity in an adaptive way, that is, when the decision variable reaches a sufficiently high degree of confidence for a particular response alternative (Servant et al., 2015). Electrophysiological and microstimulation studies in behaving monkeys have revealed that the very same neurons that encode the decision variable also encode a confidence signal (Fetsch, Kiani, Newsome, \& Shadlen, 2014; Kiani \& Shadlen, 2009). Recent behavioral and computational evidence suggest that changes of mind in decision-making and confidence are linked by a common generative mechanism (Van den Berg et al., 2016). In the drift diffusion model framework, confidence corresponds to the log posterior odds of a correct response and can be derived from the diffusion process using Bayes' rule (Kiani \& Shadlen, 2009). Our theoretical EMG bounds might correspond to a log posterior odds level.

\section{Extension to Other Conflict Tasks}

An important strength of the DMC architecture is its ability to account for behavioral data from different conflict tasks. Contrary to the Simon effect, the magnitude of flanker and Stroop compatibility effects increases over time
(Servant et al., 2014; White et al., 2011; Hübner et al., 2010; Pratte et al., 2010; but see Burle, Spieser, Servant, \& Hasbroucq, 2014). The DMC explains these distributional differences by variations in the characteristic time parameter $\tau$ of the automatic activation. Specifically, an automatic activation that develops relatively early (late) relative to the controlled activation tends to produce a decrease (increase) of the compatibility effect as RT increases (Ulrich et al., 2015). In line with this hypothesis, we found that the DMC captures the present Simon task data with an automatic activation that develops early (the peak latency of the gamma function was $\sim 30$ msec on average).

Two aspects of our electrophysiological data appear consistent with the early activation hypothesis. First, the ipsilateral negativity preceding the inhibition ramp in incompatible trials (Figure 3A, dashed rectangle) peaks $\sim 40$ msec after its onset (onset and peak latencies detected after application of a 30-Hz low-pass Butterworth filter and averaged across chroma levels). Second, partial errors are not associated with a reliable M1 inhibition (Figure 10A and $10 \mathrm{~B}$, middle). This result might suggest that the latency of partial errors is very close to the onset of the decision process. Consistent with this hypothesis, we found that partial error latencies generated by DMC extended with EMG bounds are very fast, particularly in the incompatible condition (range 39-48 msec after evidence accumulation onset; see Table 3). If the lack of M1 inhibition for partial errors in the Simon task is due to the early automatic activation, then a reliable inhibition should be observed in the flanker task. Figure 8 of Burle, Roger, Allain, Vidal, and Hasbroucq (2008; standard flanker task, EEG timelocked to the onset of partial errors) suggests that this is the case. A reanalysis of their EEG data revealed a reliable M1 inhibition associated with partial errors (one sample $t$ test against $0, t(9)=-2.4, p=.04$, data collapsed across flanker compatibility conditions). These results provide support to the general DMC framework. Combining DMC predictions with EEG and EMG measurements in other conflict tasks such as the flanker or the Stroop should provide additional insight into the relative time course of controlled and automatic activations.

\section{Conclusion}

Our model-based neuroscience study contributes to the growing body of evidence showing a representation of the decision variable in the motor system (e.g., Kelly \& O'Connell, 2013; O'Connell et al., 2012; Purcell et al., 2010, 2012; Selen et al., 2012; Donner et al., 2009; Ratcliff et al., 2003; Romo et al., 2002; Gold \& Shadlen, 2000; Hanes \& Schall, 1996), even down to the effectors (Servant et al., 2015). It also demonstrates that the decision variable is not shielded against task-irrelevant sensory information. Our findings provide new insight into the neurophysiology of human decision-making. In return, they provide support for the DMC model framework. 


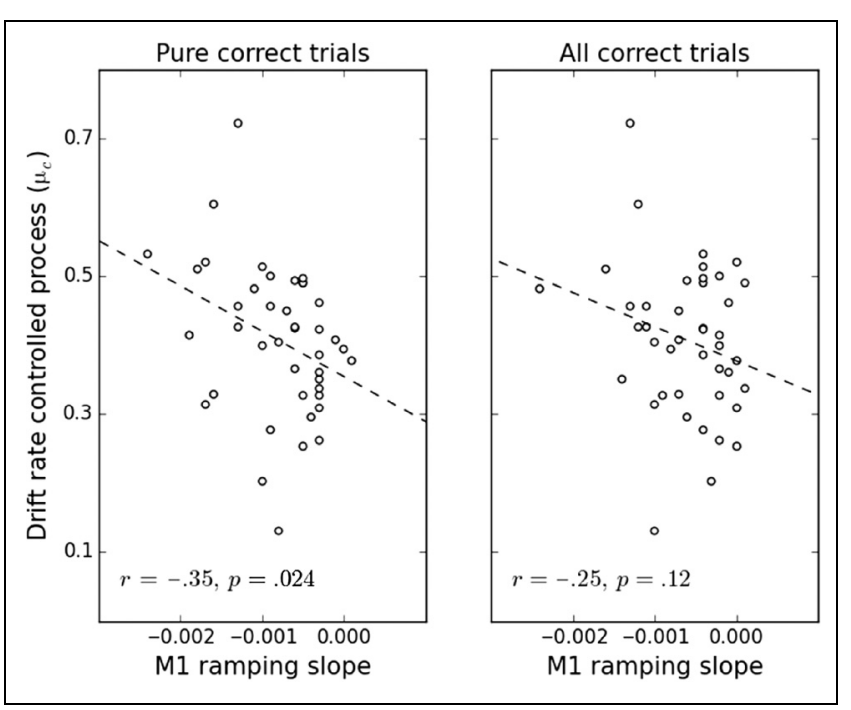

Figure A1. Intersubject correlation between the best-fitting drift rate of the controlled process $\mu_{c}$ and M1 ramping slopes of activation and inhibition EEG components for pure correct (left panel) and all correct (right panel) trials. See text for details

\section{APPENDIX A: FURTHER EVIDENCE IN FAVOR OF LINKING PROPOSITION 1}

Another (complementary) way of investigating Linking Proposition 1 consists of relating the variance in individual DMC parameter estimates with the variance of the neural signal over M1s. Specifically, one should expect a significant intersubject correlation between the drift rate of the controlled process $\mu_{c}$ and the slope of activation and inhibition EEG components. This analysis, however, is contaminated by the spillover of the automatic process to decision-making. To attenuate this contamination, we estimated ramping slopes of activation and inhibition M1 components in pure correct trials (i.e., without partial errors). Another issue concerns the high amount of noise in individual EEG data. To increase the signal-to-noise ratio, we averaged ramping slopes from compatible and incompatible trials and averaged activation and inhibition ramping slopes (multiplying inhibition slopes by -1 ), resulting in 14 (subjects) $\times 3$ (chroma levels) $=56$ data points. The correlation between M1 ramping slopes and the best-fitting drift rate of the controlled process $\mu_{c}$ in pure correct trials was significant, $r=-.35, p=.024$ (Figure A1, left). Not surprisingly, the same correlation performed in all correct trials did not reach significance $(r=-.25, p=.12$; Figure A1, right).

\section{APPENDIX B: EMPIRICAL EVIDENCE FOR A MOTOR LOCUS OF THE MN150 GENERATOR}

Figures B1 and B2 display the grand-averaged SL time courses in the six experimental conditions for stimuli presented on the right (B1) versus left (B2) side in all

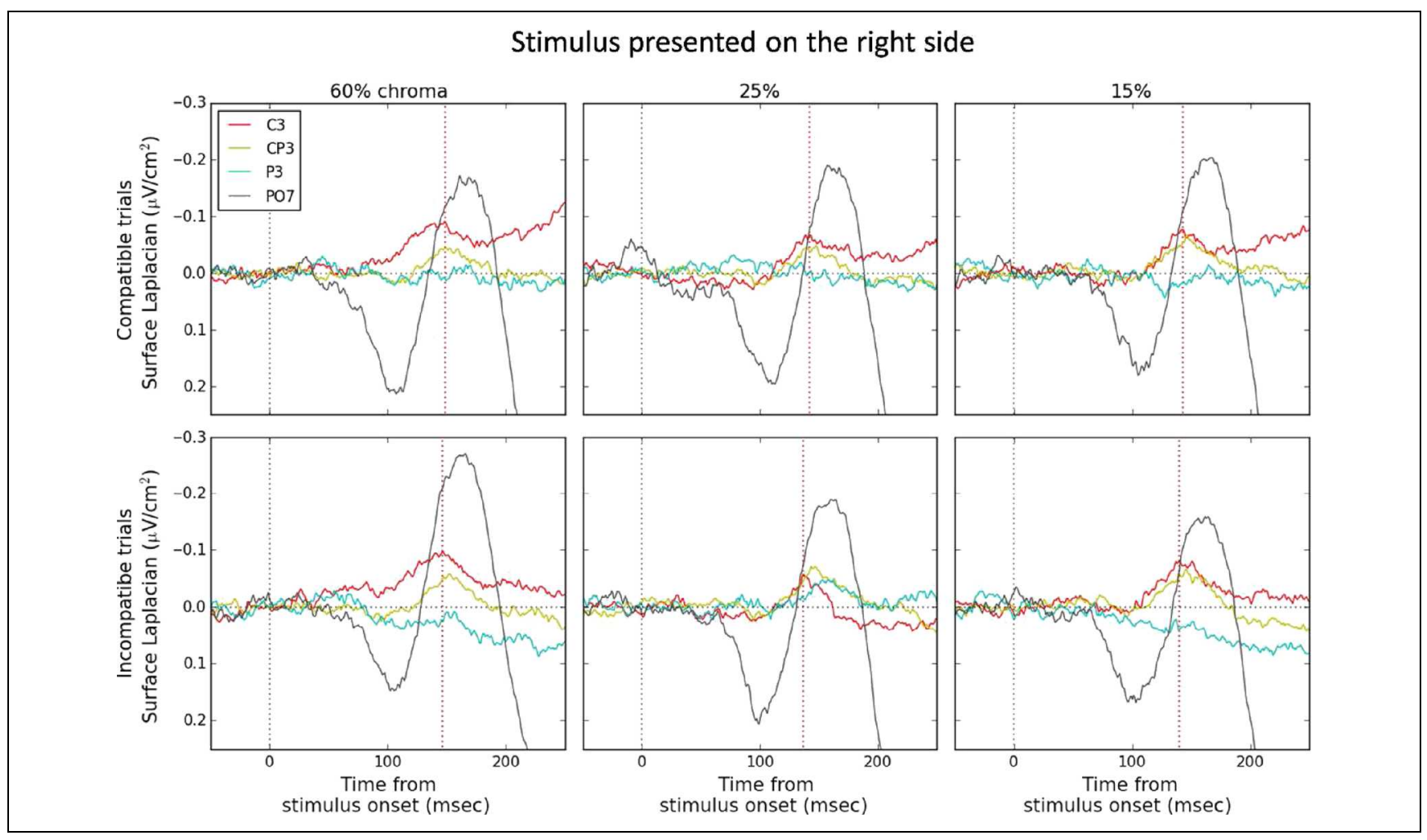

Figure B1. Grand-average SL time courses at standard sites C3, CP3, P3 and PO7 in the six experimental conditions for stimuli presented on the right side. Signals are time-locked to the onset of the stimulus and baseline-corrected -100 to 0 msec before the stimulus-locking event. 


\section{Stimulus presented on the left side}
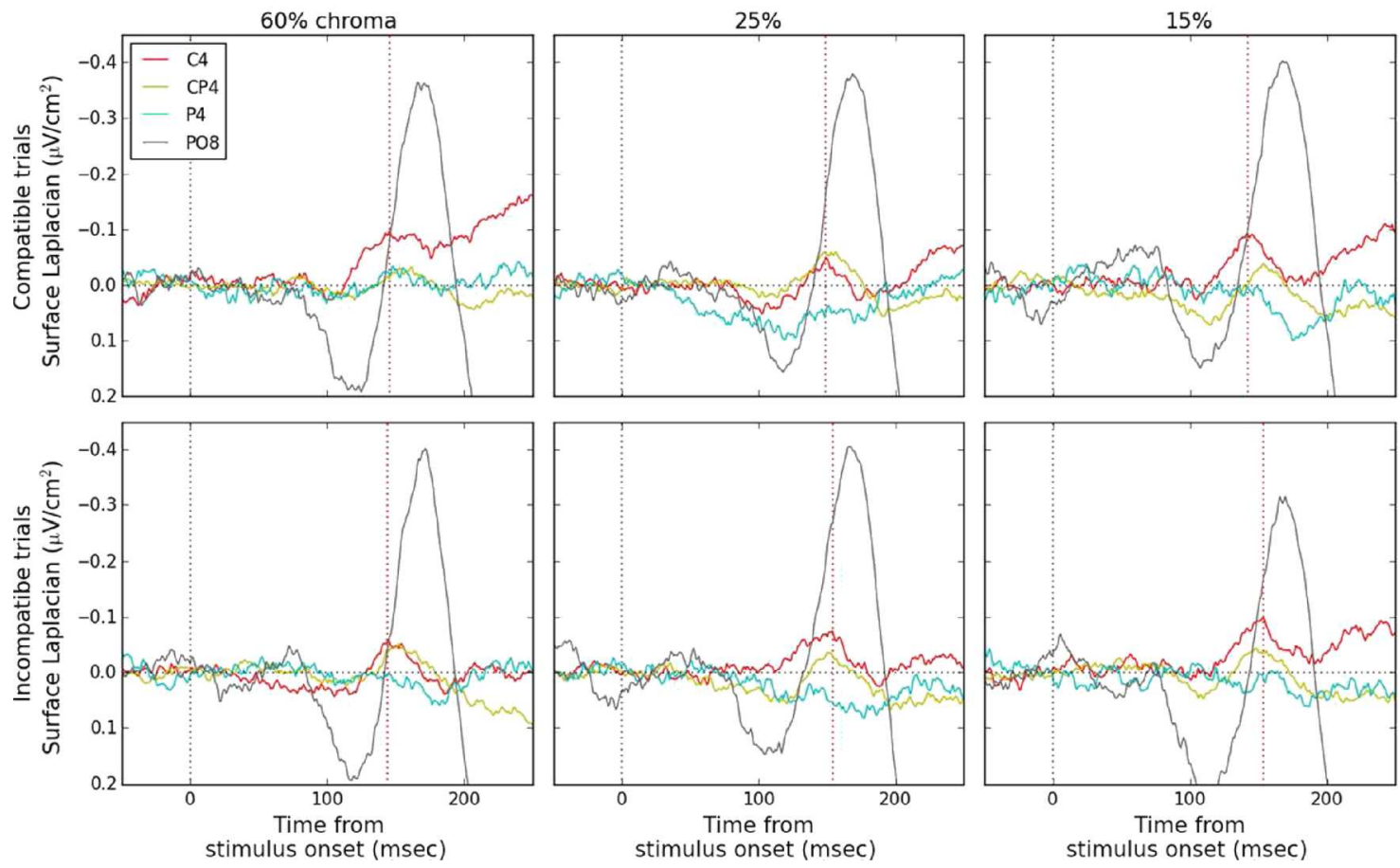

Figure B2. Grand-average SL time courses at standard sites C4, CP4, P4 and PO8 in the six experimental conditions for stimuli presented on the left side. Signals are time-locked to the onset of the stimulus and baseline-corrected -100 to $0 \mathrm{msec}$ before the stimulus-locking event.

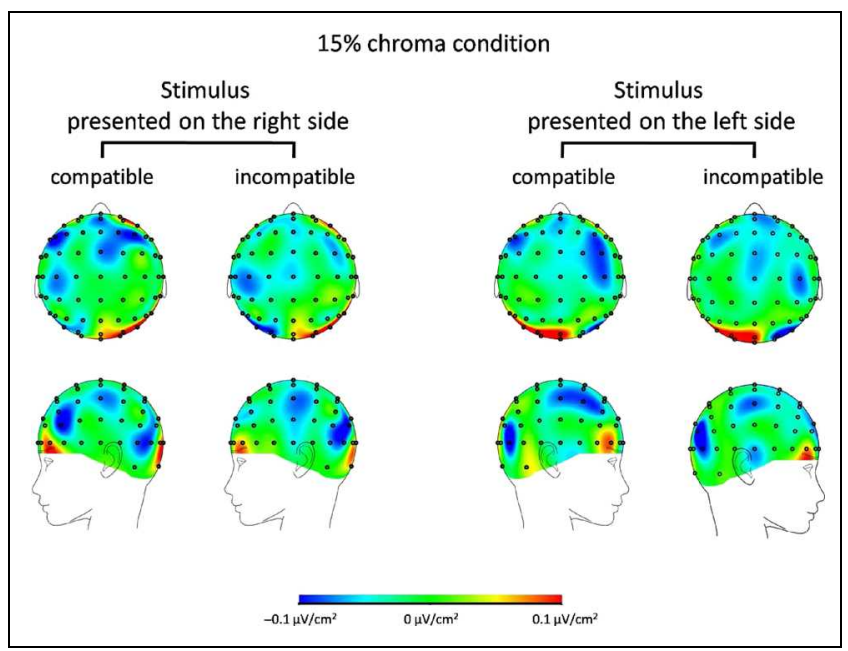

Figure B3. SL topographic maps at $150 \mathrm{msec}$ after the stimulus-locking event in the $15 \%$ chroma condition. Maps are plotted as a function of stimulus-response compatibility and stimulus side.

correct trials. Signals are time-locked to the onset of the stimulus and baseline-corrected -100 to 0 msec before the stimulus-locking event. The peak latency of the MN150 (indicated by the red dotted line) systematically precedes that of the lateralized sensory-related negativity at electrodes PO7/PO8 (all $p$ s < .05). Moreover, SL topographic maps at MN150 peak latency shown in Figure B3 clearly show a gap between motor (C3/C4) and sensory (PO7/PO8) activities. The different time courses and topographies of these activities demonstrate they are not generated by the same underlying dipole.

\section{Acknowledgments}

This research was supported by the European Research Council under the European Community's Seventh Framework Program (FP7/2007-2013 grant agreement no. 241077).

Reprint requests should be sent to Borís Burle, Laboratoire de Neurosciences Cognitives, Fédération de Recherche 3C, case C, Aix-Marseille Université, CNRS, 3 Place Victor Hugo, 13331 Marseille cedex 3, France, or via e-mail: boris.burle@univ-amu.fr.

\section{Notes}

1. The LRP is computed by subtracting electrical activities recorded over M1 ipsilateral from those over M1 contralateral to the responding hand.

2. Although the CIE LCH is considered a device-independent color space, it needs a white point reference. We chose the standard and widely used CIE D65 illuminant.

3. The DMC was also fit to behavioral data aggregated across participants (data not shown for the sake of brevity). Parameter estimates were very close to the averages of parameter estimates obtained by fitting the model to individual data.

4. Ulrich and colleagues used a beta distribution to model across-trial variability in starting point. We used a uniform distribution, as commonly done for the standard drift diffusion model (e.g., Ratcliff \& McKoon, 2008). Ratcliff (2013) showed that the model is robust to changes in the across-trial distributions of parameter values. 
5. Hereafter we will use the term surface Laplacian (SL) instead of CSD.

6. Notice the outlier contralateral activation signal in the compatible/15\% chroma condition of the $25-50 \%$ RT bin, steeper than other chroma levels. This outlier is likely responsible for the high ramping slope observed in this condition on average (Figure 3A).

7. Partial error latencies were also significantly longer in compatible compared with incompatible trials, $F(2,26)=$ $22.4, p=.0004$.

8. Decision termination bounds cannot directly correspond to a mechanical button press: some residual motor latencies are necessary to account for, for example, the corticomuscular delay. Consequently, our model extension assumes that both decision-related and non-decision-related motor components contribute to EMG activity.

9. Notice that observed and predicted partial error latencies cannot be directly compared: one would need knowledge of the duration of sensory encoding time Te that affects the partial error latencies generated by the decision accumulator. Although we previously developed a methodology to estimate Te (Servant et al., 2015), we will focus on the effects of experimental factors on partial error latencies generated by the accumulator for the sake of brevity. Comparing the observed and predicted correction times circumvents this problem, as they are calculated as the difference between two EMG onsets after sensory encoding has occurred.

10. The extended DMC slightly underestimates partial error rates in the compatible condition. Some partial EMG bursts might reflect motor noise, hence contaminating the results. These spurious bursts, however, should be evenly distributed across conditions and should not modulate the effects of our experimental variables (Hasbroucq et al., 1999, 2009).

11. The test remained nonsignificant when the data were collapsed across conditions to increase power, $t(13)=1.2$, $p=.24)$.

\section{REFERENCES}

Albantakis, L., \& Deco, G. (2009). The encoding of alternatives in multiple-choice decision making. Proceedings of the National Academy of Sciences, U.S.A., 106, 10308-10313.

Babiloni, F., Cincotti, F., Carducci, F., Rossini, P. M., \& Babiloni, C. (2001). Spatial enhancement of EEG data by surface Laplacian estimation: The use of magnetic resonance imaging-based head models. Clinical Neurophysiology, 112, 724-727.

Bogacz, R., Brown, E., Moehlis, J., Holmes, P., \& Cohen, J. D. (2006). The physics of optimal decision making: A formal analysis of models of performance in two-alternative forced-choice tasks. Psychological Review, 113, 700-765.

Burle, B., Possamai, C. A., Vidal, F., Bonnet, M., \& Hasbroucq, T. (2002). Executive control in the Simon effect: An electromyographic and distributional analysis. Psychological Research, 66, 324-336.

Burle, B., Roger, C., Allain, S., Vidal, F., \& Hasbroucq, T. (2008). Error negativity does not reflect conflict: A reappraisal of conflict monitoring and anterior cingulate cortex activity. Journal of Cognitive Neuroscience, 20, 1637-1655.

Burle, B., Spieser, L., Roger, C., Casini, L., Hasbroucq, T., \& Vidal, F. (2015). Spatial and temporal resolutions of EEG: Is it really black and white? A scalp current density view. International Journal of Psychophysiology, 97, 210-220.

Burle, B., Spieser, L., Servant, M., \& Hasbroucq, T. (2014). Distributional reaction time properties in the Eriksen task: Marked differences or hidden similarities with the Simon task? Psychonomic Bulletin E Review, 21, 1003-1010.
Burle, B., Van den Wildenberg, W. P. M., Spieser, L., \& Ridderinkhof, K. R. (in press). Preventing (impulsive) errors: Electrophysiological evidence for online inhibitory control over incorrect responses. Psychophysiology. doi:10.1111/psyp.1264.

Burle, B., Vidal, F., Tandonnet, C., \& Hasbroucq, T. (2004). Physiological evidence for response inhibition in choice reaction time tasks. Brain and Cognition, 56, 153-164.

Chatrian, G. E., Lettich, E., \& Nelson, P. L. (1988). Modified nomenclature for the "10\%" electrode system. Journal of Clinical Neurophysiology, 5, 183-186.

Churchland, A. K., Kiani, R., \& Shadlen, M. N. (2008). Decisionmaking with multiple alternatives. Nature Neuroscience, 11, 693-702.

Cisek, P., \& Kalaska, J. F. (2010). Neural mechanisms for interacting with a world full of action choices. Annual Review of Neuroscience, 33, 269-298.

Commission Internationale de l'Eclairage. 1976. ISO 116644:2008(E)/CIE S 014-4/E:2007: Joint ISO/CIE Standard: Colorimetry-Part 4: CIE $1975 L^{*} a * b *$ Colour Space.

De Clercq, W., Vergult, A., Vanrumste, B., Van Paesschen, W., \& Van Huffel, S. (2006). Canonical correlation analysis applied to remove muscle artifacts from the electroencephalogram. IEEE Transactions on Biomedical Engineering, 53, $2583-2587$.

De Jong, R., Liang, C. C., \& Lauber, E. (1994). Conditional and unconditional automaticity: A dual-process model of effects of spatial stimulus-response correspondence. Journal of Experimental Psychology: Human Perception and Performance, 20, 731-750.

Delorme, A., \& Makeig, S. (2004). EEGLAB: An open source toolbox for analysis of single-trial EEG dynamics including independent component analysis. Journal of Neuroscience Methods, 134, 9-21.

Donner, T. H., Siegel, M., Fries, P., \& Engel, A. K. (2009). Buildup of choice-predictive activity in human motor cortex during perceptual decision making. Current Biology, 19, 1581-1585.

Eriksen, C. W., Coles, M. G. H., Morris, L. R., \& O'Hara, W. P. (1985). An electromyographic examination of response competition. Bulletin of the Psychonomic Society, 23, 165-168.

Ferbert, A., Priori, A., Rothwell, J. C., Day, B. L., Colebatch, J. G., \& Marsden, C. D. (1992). Interhemispheric inhibition of the human motor cortex. Journal of Physiology, 453, $525-546$

Fetsch, C. R., Kiani, R., Newsome, W. T., \& Shadlen, M. N. (2014). Effects of cortical microstimulation on confidence in a perceptual decision. Neuron, 83, 797-804.

Gevins, A. S., Morgan, N. H., Bressler, S. L., Cutillo, B. A., White, R. M., Illes, J., et al. (1987). Human neuroelectric patterns predict performance accuracy. Science, 235, $580-585$.

Gold, J. I., \& Shadlen, M. N. (2000). Representation of a perceptual decision in developing oculomotor commands. Nature, 404, 390-394.

Gold, J. I., \& Shadlen, M. N. (2007). The neural basis of decision making. Annual Review of Neuroscience, 30, 535-574.

Gratton, G., Coles, M. G., Sirevaag, E. J., Eriksen, C. W., \& Donchin, E. (1988). Pre- and poststimulus activation of response channels: A psychophysiological analysis. Journal of Experimental Psychology: Human Perception and Performance, 14, 331-344.

Greenhouse, S., \& Geisser, S. (1959). On methods in the analysis of profile data. Psychometrika, 24, 95-112.

Hanes, D. P., \& Schall, J. D. (1996). Neural control of voluntary movement initiation. Science, 274, 427-430.

Hasbroucq, T., Burle, B., Vidal, F., \& Possamai, C. A. (2009) Stimulus-hand correspondence and direct response 
activation: An electromyographic analysis. Psychophysiology, 46, 1160-1169.

Hasbroucq, T., Possamai, C. A., Bonnet, M., \& Vidal, F. (1999). Effect of the irrelevant location of the response signal on choice reaction time: An electromyographic study in humans. Psychophysiology, 36, 522-526.

Hommel, B. (1994). Spontaneous decay of response-code activation. Psychological Research, 56, 261-268.

Hommel, B. (2011). The Simon effect as tool and heuristic. Acta Psychologica, 136, 189-202.

Hübner, R. (2014). Does attentional selectivity in global/local processing improve discretely or gradually? Frontiers in Psychology, 5, 1-11.

Hübner, R., Steinhauser, M., \& Lehle, C. (2010). A dual-stage two-phase model of selective attention. Psychological Review, 117, 759-784.

Jones, E., Oliphant, E., Peterson, P., et al. (2001). SciPy: Open source scientific tools for Python. Retrieved from www.scipy.org/

Kayser, J., \& Tenke, C. E. (2015). Issues and considerations for using the scalp surface Laplacian in EEG/ERP research: A tutorial review. International Journal of Psychophysiology, 97, 189-209.

Kelly, S. P., \& O'Connell, R. G. (2013). Internal and external influences on the rate of sensory evidence accumulation in the human brain. Journal of Neuroscience, 33, 19434-19441.

Keuken, M. C., Van Maanen, L., Bogacz, R., Schafer, A., Neumann, J., Turner, R., et al. (2015). The subthalamic nucleus during decision-making with multiple alternatives. Human Brain Mapping, 36, 4041-4052.

Kiani, R., \& Shadlen, M. N. (2009). Representation of confidence associated with a decision by neurons in the parietal cortex. Science, 324, 759-764.

Koessler, L., Maillard, L., Benhadid, A., Vignal, J. P., Felblinger, J., Vespignani, H., et al. (2009). Automated cortical projection of EEG sensors: Anatomical correlation via the international 10-10 system. Neuroimage, 46, 64-72.

Leite, F. P., \& Ratcliff, R. (2010). Modeling reaction time and accuracy of multiple-alternative decisions. Attention, Perception, \& Psychophysics, 72, 246-273.

Leuthold, H. (2011). The Simon effect in cognitive electrophysiology: A short review. Acta Psychologica (Amsterdam), 136, 203-211.

Leuthold, H., \& Schröter, H. (2006). Electrophysiological evidence for response priming and conflict regulation in the auditory Simon task. Brain Research, 1097, 167-180.

Logan, G. (1980). Attention and automaticity in Stroop and priming tasks: Theory and data. Cognitive Psychology, 12, 523-553.

Luce, R. D. (1986). Response times. New York: Oxford University Press.

Mauchly, J. W. (1940). Significance test for sphericity of a normal $n$-variate distribution. Annals of Mathematical Statistics, 11, 204-209.

Meckler, C., Allain, S., Carbonnell, L., Hasbroucq, T., Burle, B., \& Vidal, F. (2010). Motor inhibition and response expectancy: A Laplacian ERP study. Biological Psychology, 85, 386-392.

Meynier, C., Burle, B., Possamai, C. A., Vidal, F., \& Hasbroucq, T. (2009). Neural inhibition and interhemispheric connections in two-choice reaction time: A Laplacian ERP study. Psychophysiology, 46, 726-730.

O'Connell, R. G., Dockree, P. M., \& Kelly, S. P. (2012). A supramodal accumulation-to-bound signal that determines perceptual decisions in humans. Nature Neuroscience, 15, $1729-1735$.
Peirce, J. W. (2007). PsychoPy-Psychophysics software in Python. Journal of Neuroscience Methods, 162, 8-13.

Perrin, F., Bertrand, O., \& Pernier, J. (1987). Scalp current density mapping: Value and estimation from potential data. IEEE Transactions on Biomedical Engineering, 34, 283-288.

Piéron, H. (1913). Recherches sur les lois de variation des temps de latence sensorielle enfonction des intensités excitatrices. Année Psychologique, 22, 17-96.

Praamstra, P., \& Oostenveld, R. (2003). Attention and movement-related motor cortex activation: A high-density EEG study of spatial stimulus-response compatibility. Brain Research, Cognitive Brain Research, 16, 309-322.

Pratte, M. S., Rouder, J. N., Morey, R. D., \& Feng, C. (2010). Exploring the differences in distributional properties between Stroop and Simon effects using delta plots. Attention, Perception, \& Psychophysics, 72, 2013-2025.

Purcell, B. A., Heitz, R. P., Cohen, J. Y., Schall, J. D., Logan, G. D., \& Palmeri, T. J. (2010). Neurally constrained modeling of perceptual decision making. Psychological Review, 117, $1113-1143$

Purcell, B. A., Schall, J. D., Logan, G. D., \& Palmeri, T. J. (2012). From salience to saccades: Multiple-alternative gated stochastic accumulator model of visual search. Journal of Neuroscience, 32, 3433-3446.

Ratcliff, R. (1978). A theory of memory retrieval. Psychological Review, 85, 59-108.

Ratcliff, R. (2013). Parameter variability and distributional assumptions in the diffusion model. Psychological Review, 20, 281-292.

Ratcliff, R., Cherian, A., \& Segraves, M. (2003). A comparison of macaque behavior and superior colliculus neuronal activity to predictions from models of two-choice decisions. Journal of Neurophysiology, 90, 1392-1407.

Ratcliff, R., \& McKoon, G. (2008). The diffusion decision model: Theory and data for two-choice decision tasks. Neural Computation, 20, 873-922.

Ratcliff, R., \& Smith, P. L. (2004). A comparison of sequential sampling models for two-choice reaction time. Psychological Review, 111, 333-367.

Ratcliff, R., \& Starns, J. J. (2013). Modeling confidence judgments, response times, and multiple choices in decision making: Recognition memory and motion discrimination. Psychological Review, 120, 697-719.

Ridderinkhof, K. R. (2002). Activation and suppression in conflict tasks: Empirical clarification through distributional analyses. In W. Prinz \& B. Hommel (Eds.), Common mechanisms in perception and action. Attention and performance, XIX (pp. 494-519). Oxford: Oxford University Press.

Roberts, S., \& Pashler, H. (2000). How persuasive is a good fit? A comment on theory testing. Psychological Review, 107, 358-367.

Roitman, J. D., \& Shadlen, M. N. (2002). Response of neurons in the lateral intraparietal area during a combined visual discrimination reaction time task. Journal of Neuroscience, 22, 9475-9489.

Romo, R., Hernandez, A., Zainos, A., Lemus, L., \& Brody, C. D. (2002). Neuronal correlates of decision-making in secondary somatosensory cortex. Nature Neuroscience, 5, $1217-1225$.

Roswarski, T. E., \& Proctor, R. W. (2003). The role of instructions, practice, and stimulus-hand correspondence on the Simon effect. Psychological Research, 67, 43-55.

Schall, J. D. (2004). On building a bridge between brain and behavior. Annual Review of Psychology, 55, $23-50$. 
Schwarz, W., \& Miller, J. (2012). Response time models of delta plots with negative-going slopes. Psychonomic Bulletin and Review, 19, 555-574.

Selen, L. P., Shadlen, M. N., \& Wolpert, D. M. (2012). Deliberation in the motor system: Reflex gains track evolving evidence leading to a decision. Journal of Neuroscience, 32, 2276-2286.

Servant, M., Montagnini, A., \& Burle, B. (2014). Conflict tasks and the diffusion framework: Insight in model constraints based on psychological laws. Cognitive Psychology, 72, 162-195.

Servant, M., White, C., Montagnini, A., \& Burle, B. (2015). Using covert response activation to test latent assumptions of formal decision-making models in humans. Journal of Neuroscience, 35, 10371-10385.

Simon, J. R., \& Small, A. M., Jr. (1969). Processing auditory information: Interference from an irrelevant cue. Journal of Applied Psychology, 53, 433-435.

Tandonnet, C., Burle, B., Vidal, F., \& Hasbroucq, T. (2006). Knowing when to respond and the efficiency of the cortical motor command: A Laplacian ERP study. Brain Research, 1109, 158-163.

Teller, D. Y. (1984). Linking propositions. Vision Research, 24, 1233-1246.

Ulrich, R., Schröter, H., Leuthold, H., \& Birngruber, T. (2015). Automatic and controlled stimulus processing in conflict tasks: Superimposed diffusion processes and delta functions. Cognitive Psychology, 78, 148-174.

Usher, M., \& McClelland, J. L. (2004). Loss aversion and inhibition in dynamical models of multialternative choice. Psychological Review, 111, 757-769.

Valle-Inclan, F., \& Redondo, M. (1998). On the automaticity of ipsilateral response activation in the Simon effect. Psychophysiology, 35, 366-371.

Vallesi, A., Mapelli, D., Schiff, S., Amodio, P., \& Umilta, C. (2005). Horizontal and vertical Simon effect: Different underlying mechanisms? Cognition, 96, B33-B43.

Van Boxtel, G. J., Geraats, L. H., Van den Berg-Lenssen, M. M., \& Brunia, C. H. (1993). Detection of EMG onset in ERP research. Psychophysiology, 30, 405-412.

Van den Berg, R., Anandalingam, K., Zylberberg, A., Kiani, R., Shadlen, M. N., \& Wolpert, D. M. (2016). A common mechanism underlies changes of mind about decisions and confidence. Elife, 5, 1-21.

Van den Wildenberg, W. P., Wylie, S. A., Forstmann, B. U., Burle, B., Hasbroucq, T., \& Ridderinkhof, K. R. (2010). To head or to heed? Beyond the surface of selective action inhibition: A review. Frontiers in Human Neuroscience, 4, 222.

Van Maanen, L., Grasman, R. P., Forstmann, B. U., Keuken, M. C., Brown, S. D., \& Wagenmakers, E. J. (2012). Similarity and number of alternatives in the random-dot motion paradigm. Attention, Perception, \& Psychophysics, 74, 739-753.

Vidal, F., Burle, B., Grapperon, J., \& Hasbroucq, T. (2011). An ERP study of cognitive architecture and the insertion of mental processes: Donders revisited. Psychophysiology, 48, 1242-1251.

Vidal, F., Grapperon, J., Bonnet, M., \& Hasbroucq, T. (2003). The nature of unilateral motor commands in between-hand choice tasks as revealed by surface Laplacian estimation. Psychophysiology, 40, 796-805.

Wagenmakers, E.-J., \& Brown, S. (2007). On the linear relation between the mean and the standard deviation of a response time distribution. Psychological Review, 114, 830-841.

Wallace, R. J. (1971). S-R compatibility and the idea of a response code. Journal of Experimental Psychology, 88, 354-360.

Wascher, E., Schatz, U., Kuder, T., \& Verleger, R. (2001). Validity and boundary conditions of automatic response activation in the Simon task. Journal of Experimental Psychology: Human Perception and Performance, 27, 731-751.

Wascher, E., \& Wauschkuhn, B. (1996). The interaction of stimulus- and response-related processes measured by event-related lateralizations of the EEG. Electroencephalography and Clinical Neurophysiology, 99, 149-162.

White, C. N., Ratcliff, R., \& Starns, J. J. (2011). Diffusion models of the flanker task: Discrete versus gradual attentional selection. Cognitive Psychology, 63, 210-238.

Winer, B. J. (1971). Statistical principles in experimental design: Design and analysis of factorial experiments. New York: McGraw-Hill. 